\title{
Hidden Convexities in Decentralized Coordination for the Distribution Networks
}

\author{
Pirathayini Srikantha, Member, IEEE, Mithun Mallick, Student member, IEEE,
}

\begin{abstract}
The modern power grid is undergoing unprecedented levels of transformations due to the rising prevalence of diverse power entities, cyber-enablement of grid components and energy deregulations. In this paper, we focus on distribution networks (DNs) to enable the seamless plug-and-play coordination of actuating cyber-enabled power entities for costeffective and feasible system operations. The proposed distributed algorithm empowers individual cyber-physical agents residing in active power nodes with the ability to iteratively compute local actuation setpoints by exchanging information with neighbouring entities. The main contribution of this work is the identification of hidden convexities in the original non-convex optimal power flow (OPF) formulation for the DN via strategic decomposition and strong duality principles. These eliminate the need for OPF relaxations/approximations. Strong convergence and feasibility results are presented via theoretical analysis and practical simulation studies conducted on realistic systems.
\end{abstract}

Index Terms-Power System Management, Iterative Algorithms, Convex Optimization

\section{INTRODUCTION}

Climate change concerns, penetration of diverse power components and advent of innovative grid technologies are instigating widespread changes in the electricity landscape. As such, the proliferation of renewable generation systems, electric vehicles, storage devices and cyber-enablement is becoming ubiquitous in distribution networks (DNs) [1]. The DN is a low-voltage electrical infrastructure originally designed to deliver electricity from the main grid (i.e. transmission network) to power consumers. The high resistance to reactance ration associated with DN lines render it susceptible to voltage variations especially in the presence of a large number of highly-varying distributed generation (DG) systems (e.g. renewables, storage devices, etc.) and diverse loads (e.g. electric vehicles, etc.). These variations can lead to violations in system limits and therefore result in issues such as equipment damages [3], [32]. Therefore, effective DN coordination schemes that account for complex power flow constraints are vital for maintaining stable operations. The cyber-physical nature of the modern grid plays a key role in enabling this.

Existing work on grid coordination can be divided into three categories: droop-based, decentralized and centralized algorithms. With droop-based algorithms (e.g. [4] - [6]), no communication takes place amongst active power entities. Only local measurements are utilized for actuation decisions. Although these myopic decision-making structures allow for rapid response to system changes, these tend to neglect

M. Mallick and P. Srikantha are with the Department of Electrical and Computer Engineering, Western University, London, ON, Canada email: mmallic2@uwo.ca and psrikan@uwo.ca. global optimality and/or important power flow constraints. Decentralized decision-making techniques entail communication amongst active power nodes for incorporating global conditions into actuation decisions. These break-down the large complex power flow problem into smaller tractable ones that can be solved locally by participating agents [7] - [11]. Typically, difficult non-convex constraints are tackled by applying relaxations (e.g. second-order cone (SOC), semidefinite (SD)). These are exact under assumptions that may not hold under practical conditions and thereby leading to violations in system limits. Centralized algorithms involve an operator obtaining all parameters of the system and centrally computing optimal actuation for all active power entities [12] [14]. This will entail significant communication and computational overheads and hinder the flexible integration of diverse power entities into the DN. Moreover, non-convexities are either dealt with via convex relaxations or heuristic techniques that have no guarantees of optimality or convergence.

Our proposal in this paper overcomes these challenges by way of strategic decomposition of the original DN coordination problem into individual sub-problems that reveal hidden convexities. These have been uncovered using theoretical constructs that include strong duality, Schur's complement and S-procedure. Alternating direction method of multipliers (ADMM) is then applied to iteratively solve the exact convex transformations of the originally non-convex sub-problems in a decentralized manner. This can be applied to a multiagent system that is physical (i.e. actuating DN entities solve decomposed problems via information exchanges) or virtual (i.e. parallel processing capabilities of centralized computation platforms such as cloud systems and control stations). This work capitalizes on the electrical characteristics of the power system to determine strategic decompositions that eliminate non-convexities while preserving the original constraints in the problem. This differs from existing work that utilize ADMM as proposals such as [15] do not eliminate non-convexities which can result in sub-optimal solutions and other proposals such as [10] apply convex relaxations that are exact under certain assumptions. Furthermore, contributions in this paper differ from our recent work in [16] as we consider the optimal power flow problem in this paper whereas in [16] we consider a microgrid setting with linearized state variables that do not include real/reactive power variables in the dq0 frame of reference. Introducing these variables into the framework presented in [16] will introduce additional non-convexities and therefore is not suitable for the DN setting.

The contributions of this paper are six-fold and support the self-healing and adaptive smart grid vision outlined reference 
[1]: 1) A novel decentralized algorithm is proposed for the DN which empowers individual actuating entities with the ability to iteratively compute local actuation via information exchanges with neighbouring entities (e.g directly connected in the cyber plane) to seamlessly adapt to changes in the DN; 2) This algorithm can also be applied in a centralized setting in a server composed of multiple parallel processing units to efficiently compute actuation that accounts for difficult constraints in planning processes; 3) The original non-convex optimal coordination problem for the DN is decomposed with the introduction of auxiliary variables and transformed into convex subproblems using the Schur's complement; 4) Strong duality gap of the convex problem is established via the S-procedure and conditions for the convergence of the iterative algorithm to the Karush Kuhn Tucker (KKT) point of the original coordination problem are derived; 4) As strong duality gap is established, there is no need to apply relaxations/approximations to the original non-convex coordination problem; 5) The proposed algorithm for balanced DNs is extended to unbalanced multiphase DNs; and 6) Comprehensive simulation and comparative studies are conducted on MATLAB and MatPower platforms to demonstrate practical viability of the theoretical results established earlier.

As such, the remainder of this paper is organized as follows. In Sec. II, the cyber-physical attributes of the system model are presented along with the optimal DN coordination problem. The proposed algorithm along with theoretical studies for balanced DNs are presented in Sec. III. This is then extended to unbalanced multi-phase DNs in Sec. IV. Then, comprehensive practical simulations and comparative studies are conducted in Sec. V. Finally, the paper is concluded in Sec. VI.

\section{SySTEM MODEL}

In this section, we present the cyber and physical models of the DN utilized to design the proposed coordination algorithm. The system model presented in this section is based on the smart grid vision (e.g. [1]) which is composed of ubiquitous computational and communication capabilities in active and passive power elements residing in the DN. However, this smart grid vision has not yet been realized in many DNs today and will become a reality in the near future. In these cases, the electric power utility will be able to apply the proposed algorithm to perform computations on a central server composed of parallel cores. Each core can perform the task of a bus agent and iteratively compute the solutions of the sub-problems as proposed in the algorithm presented in this paper. This divide-and-conquer approach allows for the efficient use of utility resources. Hence, the proposed distributed model can be readily translated into a central paradigm that organizes parallel computations of the solution in an efficient manner.

As such, we focus on the distributed paradigm and present the cyber-physical interactions between actuating power components in the DN in Fig. 1. Then, the optimal DN coordination problem is formulated and the associated challenges with directly solving it are highlighted.

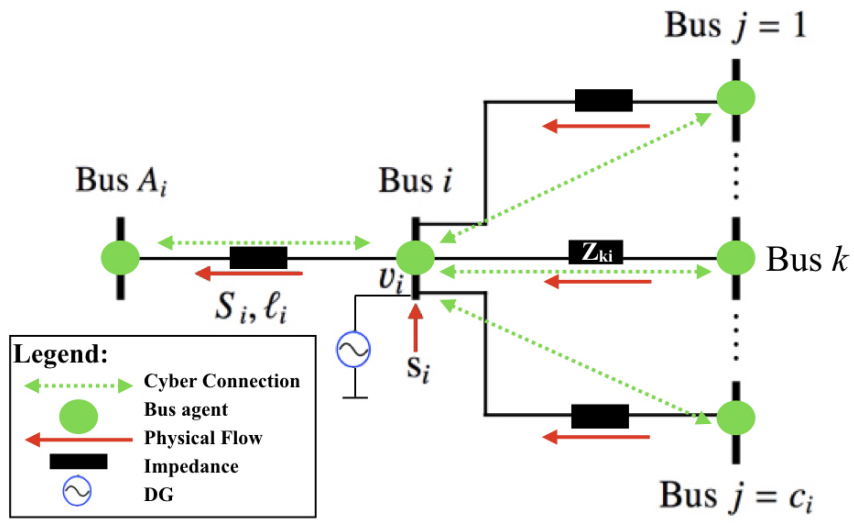

Fig. 1: Cyber-physical interactions

\section{A. Cyber Model}

Traditionally passive power devices are now equipped with the ability to communicate with one another, gather sensor measurement data and perform local computations/actuations [1]. In the cyber model considered in this paper, each bus in the DN will be composed of an intelligent module (referred to as bus agent) that can communicate with neighbouring buses (i.e. separated by a single electrical line), perform local computations and actuate locally connected power sources ( (e.g. storage, renewables, etc.)). Power sources can be dispatchable and undispatchable (e.g. photovoltaics). Undispatchable sources can be coupled with storage systems which can be actuated (e.g. reference [30]). This way, the bus agent can control the power injected by undispatchable sources via the coupled storage element. This is a general approach based on the smart grid vision that accommodates power injecting entities at every bus. However, in today's DNs, a small subset of buses consist of power injecting elements. In this case, methods such as Kron-reduction can be utilized to simplify the system model around the active buses and thus limit the cyber model being used [18]. Equivalent line and load parameters can be utilized in the computation and data exchange process of the proposed algorithm. This will be investigated in future work.

As such, in the general framework presented in this paper, each bus agent will have access to local sensors that provide local state information such as bus voltage, currents, etc. Devices such as the micro-synchrophasors are being designed specifically for the DN at lower costs than traditional PMUs that are deployed in the transmission network. These facilitate greater system awareness and allow for distributed computations by actuating DN agents [19]. Cyber interactions between bus agents are illustrated via the dotted green arrows in Fig. 1. Each bus agent will exchange information with neighbouring agents every $30 \mathrm{~ms}$ via wireless communication channels supported by ZigBee which is a commonly deployed protocol in the smart grid [17]. As the delay in single-hop wireless communication is typically estimated to be between 8 to $20 \mathrm{~ms}$, this information exchange frequency accounts for possible delays in practical systems [23]. The proposed algorithm performs coordination every $9 \mathrm{~s}$. Power demands 
and generation within the $\mathrm{DN}$ are assumed to be constant over this $9 \mathrm{~s}$ interval (e.g. steady-state coordination). Forecast models proposed in the recent literature can predict generation and demands over these short intervals with high accuracies [24]. This coordination interval is much shorter than the typical one-hour or day-ahead horizons utilized by system operators. Forecast models applied to predict renewable generation outputs over longer coordination horizons are associated with high error margins [24]. Our algorithm avoids this issue by performing coordination at high granularity and utilize accurate representations of system resources and limits.

It is possible for the information exchanged amongst agents to be lost. The consensus relations established between variables maintained by individual agents (this is presented later in Sec. III) ensure that other agents are able to sense these discrepancies and recover through subsequent iterations. There also exist privacy and security concerns for data being exchanged amongst agents. Standard cryptographic techniques available in the wireless communication protocols (e.g. Advanced Encryption Standard (AES)) can be applied to protect the information exchanged [20]. These will not impose significant overheads as the information exchanged is lightweight. As future work, we intend to explore alternate security mechanisms (e.g. differential privacy) that can be leveraged to secure data being exchanged.

The proposed algorithm decomposes the original OPF problem into sub-problems that can be solved by actuating agents via information exchanges with neighbouring nodes. Thus, this naturally fits the smart grid vision where the power grid is equipped with advanced communication, measurement and control systems [1]. With pressing concerns regarding climate change and grid reliability, the modern power system evolving steadily towards this smart grid vision. Smart grid prototypes such as EcoGrid showcase the far-fetching benefits of this vision [21]. One example is the widespread implementation of the advanced metering infrastructure which is composed of smart meters that are capable of recording local measurements (e.g. real/reactive power, current, voltage, etc.) at granularities ranging from every minute to every hour [22].

Our algorithm also applies in a centralized setting which is equipped with parallel processing units that are each assigned a decomposed sub-problem. A system operator such as the electric power utility can forecast/predict the loads and generation capacities in the DN and supply these parameters to the proposed algorithm which will then compute the solutions while accounting for non-convexities.

\section{B. Physical Model}

Physical electrical couplings amongst bus agents will be modelled via graph theoretic notations. The DN is an electrical network that can be represented as a graph $G$ that is composed of bus agents that belong to the set $\mathcal{N}$ and electrical lines forming the directed set $\vec{E}$. Line $(i \rightarrow j) \in \vec{E}$ directly connects bus $i \in \mathcal{N}$ to another bus $j \in \mathcal{N}$. We adopt the branch flow model introduced in [7] where the physical flows (e.g. power and current) along the power lines are assumed to be directed towards the feeder bus. This physical model pertains to balanced DNs which is utilized to introduce the proposed algorithm via simpler notations. Then, in Sec. IV, we extend the proposal to unbalanced DN systems. The feeder bus is denoted as bus 0 . Since the $\mathrm{DN}$ is a radial network (composed of no cycles), each bus agent $i \in \mathcal{N} / 0$ will have a unique ancestor $A_{i}$ (i.e. $A_{i}$ is the bus agent closer to the feeder bus and separated by a single line from bus $i$ ). These electrical flows are represented by solid red uni-directional arrows in Fig. 1.

Each power line $(i \rightarrow j) \in \vec{E}$ has complex impedance $z_{i j}=r_{i j}+\mathbf{j} x_{i j}$ where $\mathbf{j}$ is the imaginary number. Each bus agent $i \in \mathcal{N} / 0$ is associated with the following complex state variables: bus voltage $V_{i}$, net power injection in the bus $s_{i}$, power flow $S_{i A_{i}}$ along the line $\left(i \rightarrow A_{i}\right)$, and the corresponding current flow $I_{i A_{i}}$. It is assumed that there exists sufficient flexible power devices in each bus $i$ to absorb surplus generation from uncontrollable renewable energy sources so that these can be coordinated to prevent over-voltage conditions in the DN. Since the physical flows in the network are assumed to be directed towards the feeder bus and each bus will have a unique ancestor due to the radial structure of the DN, the second subscript in variables representing power and current flows along the line $\left(i \rightarrow A_{i}\right) \in \vec{E}$ will be removed and be denoted as $S_{i}$ and $I_{i}$ respectively. The feeder bus will maintain constant bus voltage of 1 p.u. and inject real/reactive power (obtained from the main grid) into the DN as necessary when available generation capacity in the DN is not sufficient to meet local demands. Steady-state power balance relations can now be expressed in terms of these parameters and variables as follows:

$$
\begin{aligned}
S_{i} & =\sum_{j \in C_{i}}\left(S_{j}-z_{j i}\left|I_{j}\right|^{2}\right)+\mathrm{s}_{i}, & & i \in \mathcal{N} \\
I_{i} & =\left(V_{i}-V_{A_{i}}\right) / z_{i A_{i}}, & & i \in \mathcal{N} / 0 \\
S_{i} & =V_{i} I_{i}^{H}, & & i \in \mathcal{N} / 0
\end{aligned}
$$

Eq. 1 represents power balance dictated by the Tellegen's theorem which necessitates that the sum of all power flows into bus $i$ must be 0 . Power flow $S_{j}$ that originates at bus $j$ and enters into bus $A_{j}$ via the line $\left(j \rightarrow A_{j}\right) \in \vec{E}$ is subject to line losses $z_{j i}\left|I_{j}\right|^{2}$. These incoming power flows from all the descendent/children buses of bus $i$ forming the set $C_{i}$ along with bus power injection $s_{i}$ must be equal to the power flowing out of bus $i$ via line $i \rightarrow A_{i}$. $S_{0}$ is considered to be 0 for the feeder bus as it has no ancestors. Eq. 2 relates current flow $I_{i}$ on line $i \rightarrow A_{i}$ with the corresponding voltage drop $V_{i}-V_{A_{i}}$. Eq. 3 relates power flow with voltage and current where $H$ is the complex conjugate operator. As the feeder bus serves as the root node of the DN and hence has no ancestors, Eqs. 2 and 3 do not apply to this bus.

Eqs. 1 to 3 are complex relations. As it is easier to work with real variables, real and imaginary components in these relations can be considered separately. However, this will result in the introduction of more non-linear terms (e.g. cosine, etc.) and cause further complications. To avoid these issues, Eqs. 1 to 3 are manipulated so that only the magnitudes of the complex state variables are considered [7]. For this, the following variables are introduced: $\ell_{i}=\left|I_{i}\right|^{2}$ and $v_{i}=\left|V_{i}\right|^{2}$ 
which represent the square magnitude of the complex current and voltage variables respectively. Bus power injection and line power flow can be represented by the real and reactive power components: $s_{i}=p_{i}+\mathbf{j} q_{i}$ and $S_{i}=P_{i}+\mathbf{j} Q_{i}$. Now, applying the variable transformations, substituting Eq. 3 into Eq. 2 and multiplying Eq. 3 with the complex conjugate of itself results in:

$$
\begin{aligned}
& P_{i}=\sum_{j \in C_{i}}\left(P_{j}-r_{j i} \ell_{j}\right)+p_{i}, \quad i \in \mathcal{N} \\
& Q_{i}=\sum_{j \in C_{i}}\left(Q_{j}-x_{j i} \ell_{j}\right)+q_{i}, \quad i \in \mathcal{N} \\
& v_{i}=v_{A_{i}}+2\left(r_{i A_{i}} P_{i}+x_{i A_{i}} Q_{i}\right)-\left(r_{i A_{i}}^{2}+x_{i A_{i}}^{2}\right) \ell_{i}, i \in \mathcal{N} / 0 \\
& v_{i} \ell_{i}=P_{i}^{2}+Q_{i}^{2}, i \in \mathcal{N} / 0
\end{aligned}
$$

The above set of relations are now composed of only real variables. Since only the magnitudes of variables are considered, the phase angles are not included in these relations. According to reference [7], phase angles can be recovered for system states belonging to radial systems like the DN.

Eqs. 4 to 7 dictate power flow relations resulting from the electrical couplings in the DN. Next, limits on DN variables are defined for the stable and feasible operations in the DN. As such, the voltage magnitude at each bus $i \in \mathcal{N}$ must remain within a certain threshold of the nominal value of 1 p.u. to prevent equipment damages [3]:

$$
\underline{V}^{2} \leq v_{i} \leq \bar{V}^{2}, i \in \mathcal{N}
$$

where the upper and lower bounds on the voltage magnitude are $\underline{V}$ and $\bar{V}$ respectively. The next constraint is imposed on the net power injected into each bus which is governed by the generation capacities of local sources:

$$
\begin{aligned}
& \mathrm{s}_{i}=s_{G i}-\mathrm{s}_{L i}, i \in \mathcal{N} \\
& s_{G i} \leq s_{G i} \leq \overline{s_{G i}}, i \in \mathcal{N} \\
& \ell_{i} \leq \bar{\ell}_{i}, i \in \mathcal{N} / 0 \\
& P_{i} \leq \bar{P}_{i}, i \in \mathcal{N} / 0
\end{aligned}
$$

Eq. 9 sets the net power injection to be the overall complex power generation $s_{G i}$ in bus $i$ minus the complex power load $s_{L i}$. Eq. 10 imposes upper and lower limits on the power generated in bus $i$ based on local generation capacities. The lower limits ensure that the power injected by uncontrollable sources are also accounted for in the proposed algorithm. In Eq. 11, we have included an upper limit on the magnitude of current flow on each line $\left(i \rightarrow A_{i}\right)$ in the DN. The next constraint imposes an upper limit on reverse flow (the direction of power flow assumed in this paper is towards the feeder) over line $\left(i \rightarrow A_{i}\right)$ which is necessary in the presence of transformers.

We next consider discrete control devices such as online load tap changers (OLTCs) and shunt devices. An OLTC is associated with tap ratio $t_{l}$ that can typically take one of 33 discrete values where $l \in \mathcal{L}$ which denotes all lines that contain these OLTCs in the DN [8]. These values range from $[0.9 \ldots 1.1]$ with an increment of $5 / 8 \%$ p.u. Thus, the tap ratio is subject to the following constraint:

$$
t_{l} \in\{0.9,0.90625, \ldots, 1.09375,1.1\}
$$

The effect of the tap ratio of the OLTC residing in line $l=$ $(i \rightarrow j)$ is:

$$
\frac{1}{t_{l}^{2}} v_{i}-v_{j}=2\left(r_{l} p_{i j}+x_{l} q_{i j}\right)-\left(r_{l}^{2}+x_{l}^{2}\right) \ell_{i}
$$

It is clear that the above relation is non-convex as it contains a quadratic term and one of the variables (i.e. $t_{l}$ takes value in a discrete set). Shunt devices are also discrete control devices with non-convex properties like the OLTC. The method by which we decompose the problem for OLTCs can also be applied for shunt devices.

Hence, the electrical couplings and physical system limits defined in Eqs. 4 to 14 dictate the actuation of active power components in the DN.

\section{Optimal DN Coordination Problem}

Now, based on the cyber and physical couplings in the DN, the optimal DN coordination problem is formulated in $\mathcal{P}_{O C}$ :

$$
\begin{aligned}
& \mathcal{P}_{O C}: \min _{S_{i}, s_{i}, v_{i}, \ell_{i}} \sum_{i \in \mathcal{N}} f_{i}\left(S_{i}, s_{i}, v_{i}, \ell_{i}\right) \\
& \text { subject to : Eqs. } 4-14
\end{aligned}
$$

The objective $f_{i}$ is a linear combination of cost associated with each bus agent which is a function of local state variables (e.g. cost of injecting power by local sources, power losses which are functions of current, etc.). This function is typically convex as it is either linear or consists of quadratic terms with positive coefficients [25]. Eqs. 4-6 and Eqs. 8-10 are linear relations and these form convex sets. However, Eq. 7 is a quadratic equality constraint and thus is not a convex set. Similarly, the constraints introduced by discrete control devices such as Eq. 13 and 14 are also non-convex. Optimization problems composed of non-convex objectives/constraints are typically NP-Hard to solve and become intractable as the variable space becomes large. In existing literature, this non-convexity has been dealt with via relaxation techniques (e.g. second-order cone, semi-definite relaxations and/or linearizations). Under certain assumptions, relaxations such as SOC and SD are proven to be exact in radial networks [25]. However, these assumptions are limiting and can result in infeasibilities of system limits when violated. In this paper, we capitalize on the cyber enablement of the power grid to strategically decompose $\mathcal{P}_{O C}$ into sub-problems that can be solved by each bus agent. These sub-problems are transformed into equivalent convex problems for which exact solutions can be computed. This decomposition and transformation process is presented in detail in the following section.

\section{PROPOSAL}

In this section, we propose a decentralized coordination algorithm to solve $\mathcal{P}_{O C}$. We first introduce auxiliary variables called the perspective variables for each bus agent to decompose the problem into individual sub-problems that can be solved locally. These sub-problems are of two different 
compositions: Type 1) Convex objective and several linear constraints and Type 2) Convex objective and one non-convex quadratic constraint. The first problem can be solved directly due to its convex nature. The second problem, however, is not convex. To overcome this issue, we leverage on Schur's Complement and S-procedure to show that strong duality holds for the non-convex sub-problem and this is leveraged to identify the convex equivalent of the Type 2 problem. Thus, the exact solution for this sub-problem can also be computed. Then, ADMM is leveraged to establish consensus between the actual and auxiliary variables amongst bus agents across the entire system via information exchanges with neighbouring power entities. These steps are detailed in the following.

\section{A. Problem Decomposition}

The optimal DN coordination problem $\mathcal{P}_{O C}$ consists of constraints (i.e. Eqs. 4 to 6) for each bus $i$ that depend on variables that belong to immediate neighbours (i.e. children $j \in C_{i}$ and ancestor $A_{i}$ ). Due to these interdependencies, $\mathcal{P}_{O C}$ cannot be directly decomposed. In a manner similar to reference [10], we introduce auxiliary variables (referred to as the perspective variables in this paper) to maintain local copies of variables belonging to neighbouring bus agents. Thus, the problem can now be decoupled for each bus agent based on these perspective variables. However, consensus must be established between the actual and perspective variables to maintain equivalence with the original problem $\mathcal{P}_{O C}$.

For this, two sets of variables maintained by each bus agent $i$ are first defined. Local state variables (e.g. voltage, power flow, current) form the set $x_{i}$. The perspective variables which are local copies of state variables belonging to neighbouring bus agents form the set $y_{i}$. For instance, the perspective of $v_{j}$ which is a variable belonging to bus agent $j$ from the point of view of bus agent $i$ is maintained in $v_{j i}^{y}$. First and second subscripts in a perspective variable denote the perspective of and perspective from respectively. Based on the problem formulation presented in $\mathcal{P}_{O C}$, each bus agent $i$ will maintain the following local $x_{i}$ and perspective $y_{i}$ variables:

$$
\begin{aligned}
& x_{i}=\left\{P_{i}^{x}, Q_{i}^{x}, v_{i}^{x}, \ell_{i}^{x}, p_{i}^{x}, q_{i}^{x}\right\} \\
& y_{i}=\left\{P_{i i}^{y}, Q_{i i}^{y}, v_{i i}^{y}, \ell_{i i}^{y}, p_{i i}^{y}, q_{i i}^{y}, v_{A_{i} i}^{y}, P_{j i}^{y}, Q_{j i}^{y}, \ell_{i i}^{y} \forall j \in C_{i}\right\}
\end{aligned}
$$

These local and perspective variables are subject to constraints listed in Eqs. 4 to 12 and these define the feasible regions: $x_{i} \in \mathcal{X}_{i}$ and $y_{i} \in \mathcal{Y}_{i}$. The constraints are divided so that all the constraints containing variables associated with the neighbouring bus agents and/or are convex form $\mathcal{Y}_{i}$ and the non-convex constraint forms $\mathcal{X}_{i}$. The only non-convex constraint in $\mathcal{P}_{O C}$ is Eq. 7. This constraint can be equivalently expressed as:

$$
\begin{aligned}
& v_{i} \ell_{i} \geq P_{i}^{2}+Q_{i}^{2}, i \in \mathcal{N} / 0 \\
& v_{i} \ell_{i} \leq P_{i}^{2}+Q_{i}^{2}, i \in \mathcal{N} / 0
\end{aligned}
$$

Eq. 15 is convex (i.e. it is a second-order cone) while Eq. 16 is non-convex. These two constraints are split between the $\mathcal{X}_{i}$ and $\mathcal{Y}_{i}$ sets as follows.

$$
\begin{aligned}
\mathcal{X}_{i}=\left\{x_{i} \mid v_{i}^{x} \ell_{i}^{x} \leq\left(P_{i}^{x}\right)^{2}+\left(Q_{i}^{x}\right)^{2}\right\} & \\
\mathcal{Y}_{i}=\left\{y_{i} \mid P_{i}^{y}\right. & =\sum_{j \in C_{i}}\left(P_{j}^{y}-r_{j i} \ell_{j}^{y}\right)+p_{i}^{y}, \\
Q_{i}^{y} & =\sum_{j \in C_{i}}\left(Q_{j}^{y}-x_{j i} \ell_{j}^{y}\right)+q_{i}^{y}, \\
v_{i}^{y} & =v_{A_{i}}^{y}+2\left(r_{i A_{i}} P_{i}^{y}+x_{i A_{i}} Q_{i A_{i}}^{y}\right)-\left(r_{i A_{i}}^{2}+x_{i A_{i}}^{2}\right) \ell_{i}^{y}, \\
v_{i}^{y} \ell_{i}^{y} & \left.\leq\left(P_{i}^{y}\right)^{2}+\left(Q_{i}^{y}\right)^{2}\right\}
\end{aligned}
$$

Thus, $\mathcal{X}_{i}$ is composed of $\mathrm{x}$-variables and a non-convex constraint while $\mathcal{Y}_{i}$ is composed of y-variables and convex constraints. As constraint sets $\mathcal{X}_{i}$ and $\mathcal{Y}_{i}$ contain only local variables, these can be solved independently by bus agents. However, the perspective variables maintained by each bus agent must be equal to the actual variables of the neighbouring agents. To account for this, problem $\mathcal{P}_{D}$ is formulated where consensus constraints are added. $\mathcal{P}_{D}$ is therefore an equivalent reformulation of $\mathcal{P}_{O C}$ :

$$
\begin{aligned}
& \mathcal{P}_{D}: \underset{x_{i} \in \mathcal{X}_{i}, y_{i} \in \mathcal{Y}_{i}}{\operatorname{minimize}} \sum_{i \in \mathcal{N}} f_{i}\left(x_{i}\right) \\
& \text { subject to: } \forall i \in \mathcal{N} \\
& P_{i}^{x}=P_{i, i}^{y}, Q_{i}^{x}=Q_{i, i}^{y}, v_{i}^{x}=v_{i, i}^{y}, \ell_{i}^{x}=\ell_{i, i}^{y}, p_{i}^{x}=p_{i, i}^{y}, q_{i}^{x}=q_{i, i}^{y}, \\
& P_{i}^{x}=P_{i, A i}^{y}, Q_{i}^{x}=Q_{i, A i}^{y}, \ell_{i}^{x}=\ell_{i, A i}^{y}, v_{i}^{x}=v_{i, j}^{y} \forall j \in C_{i}
\end{aligned}
$$

It is clear that $\mathcal{P}_{D}$ is separable for each bus agent $i$ in terms of the $x_{i}$ and $y_{i}$ variables without the consensus constraints. Let the consensus constraints be represented as $M x=N y$ where $M$ and $N$ are constant matrices. The augmented Lagrangian $\mathcal{L}_{\rho}$ of $\mathcal{P}_{D}$ is:

$$
\begin{aligned}
& \mathcal{L}_{\rho}(x, y, \nu)=\sum_{i \in \mathcal{N}} f_{i}\left(x_{i}\right)+\nu^{T}(M x-N y)+\frac{\rho}{2}\|M x-N y\|_{2}^{2} \\
& =\sum_{i \in \mathcal{N}}\left\{f_{i}\left(p_{i}^{x}\right)+\nu_{i i}^{P}\left(P_{i}^{x}-P_{i i}^{y}\right)+\nu_{i i}^{Q}\left(Q_{i}^{x}-Q_{i i}^{y}\right)+\nu_{i i}^{y}\left(v_{i}^{x}-v_{i i}^{y}\right)\right. \\
& +\nu_{i i}^{\ell}\left(\ell_{i}^{x}-\ell_{i i}^{y}\right)+\nu_{i i}^{p}\left(p_{i}^{x}-p_{i i}^{y}\right)+\nu_{i i}^{q}\left(q_{i}^{x}-q_{i i}^{y}\right) \\
& +\sum_{\forall j \in C_{i}}\left(\nu_{j i}^{P}\left(P_{j}^{x}-P_{j i}^{y}\right)+\nu_{j i}^{Q}\left(Q_{j}^{x}-Q_{j i}^{y}\right)+\nu_{j i}^{\ell}\left(\ell_{j}^{x}-\ell_{j i}^{y}\right)\right) \\
& +\nu_{A i i}^{v}\left(v_{A i}^{x}-v_{A i i}^{y}\right)+\frac{\rho}{2}\left(\left(P_{i}^{x}-P_{i i}^{y}\right)^{2}+\left(Q_{i}^{x}-Q_{i i}^{y}\right)^{2}+\left(v_{A i}^{x}-v_{A i i}^{y}\right)^{2}\right. \\
& \left.+\left(v_{i}^{x}-v_{i i}^{y}\right)^{2}+\left(\ell_{i}^{x}-\ell_{i i}^{y}\right)^{2}+\left(\mathrm{p}_{i}^{x}-\mathrm{p}_{i i}^{y}\right)^{2}+\left(\mathrm{q}_{i}^{x}-\mathrm{q}_{i i}^{y}\right)^{2}\right) \\
& \left.+\sum_{\forall j \in C_{i}} \frac{\rho}{2}\left(\left(P_{j}^{x}-P_{j i}^{y}\right)^{2}+\left(Q_{j}^{x}-Q_{j i}^{y}\right)^{2}+\left(\ell_{j}^{x}-\ell_{j i}^{y}\right)^{2}\right)\right\}
\end{aligned}
$$

where $\nu$ is the dual variable associated with the consensus constraints and the augmented term $\frac{\rho}{2}\|M x-N y\|_{2}^{2}$ enforces strict convexity for $\rho>0$. The dual $\mathcal{P}_{D}$ is:

$$
\mathcal{P}_{D}^{\text {dual }}: \underset{\nu}{\operatorname{maximize}} \underset{x \in \mathcal{X}, y \in \mathcal{Y}}{\operatorname{minimize}} \mathcal{L}_{\rho}(x, y, \nu)
$$

ADMM is leveraged to solve this problem iteratively where every bus agent sequentially computes $x_{i}^{k+1}, y_{i}^{k+1}$, and $\nu_{i}^{k+1}$ at each iteration $k$ :

$$
\begin{aligned}
x_{i}^{k+1} & =\underset{x_{i} \in \mathcal{X}_{i}}{\operatorname{argmin}} \mathcal{L}_{p}\left(x, y^{k}, \nu^{k}\right) \\
y_{i}^{k+1} & =\underset{y_{i} \in \mathcal{Y}_{i}}{\operatorname{argmin}} \mathcal{L}_{p}\left(x^{k+1}, y, \nu^{k}\right) \\
\nu_{i}^{k+1} & =\nu_{i}^{k}+\rho\left(M x^{k+1}-N y^{k+1}\right)
\end{aligned}
$$


When bus agent $i$ solves $U 1$ to obtain $x_{i}^{k+1}$, all terms in $\mathcal{L}_{\rho}$ not containing $x_{i}$ become constants and are therefore ignored. The remaining terms are composed of $x_{i}$ variables having coefficients that belong to $y^{k}$ and/or $\nu^{k}$ (due to quadratic terms in Eq. 17) which are variables maintained by neighbouring bus agents. In $U 1$, these are fixed to the values computed in the previous iteration which are obtained from neighbouring bus agents via single hop communication at the end of the previous iteration. Once, $x_{i}^{k+1}$ is computed, this is exchanged with neighbouring agents as this will be required for the computation of $y_{k+1}^{i}$. Then, when $y_{k+1}^{i}$ is computed, these values are exchanged with neighbouring agents to facilitate the computation of $\nu_{i}^{k+1}$. This process is repeated until the residual $\|M x-N y\|_{2}^{2} \leq \epsilon$ where $\epsilon$ is a very small positive value. This information exchange process is illustrated in Fig. 3. When $\rho$ is strictly positive and the $\mathrm{x}$ and $\mathrm{y}$ sub-problems are convex, convergence is guaranteed as shown in reference [26]. The value selected for $\rho$ will affect the convergence time. This parameter is typically selected empirically [26]. We have selected $\rho$ to be 100 which allows for reasonable convergence speed for all the systems (33-bus, 64-bus and 136bus) considered as detailed in Sec. V as illustrated in Figure 2 .

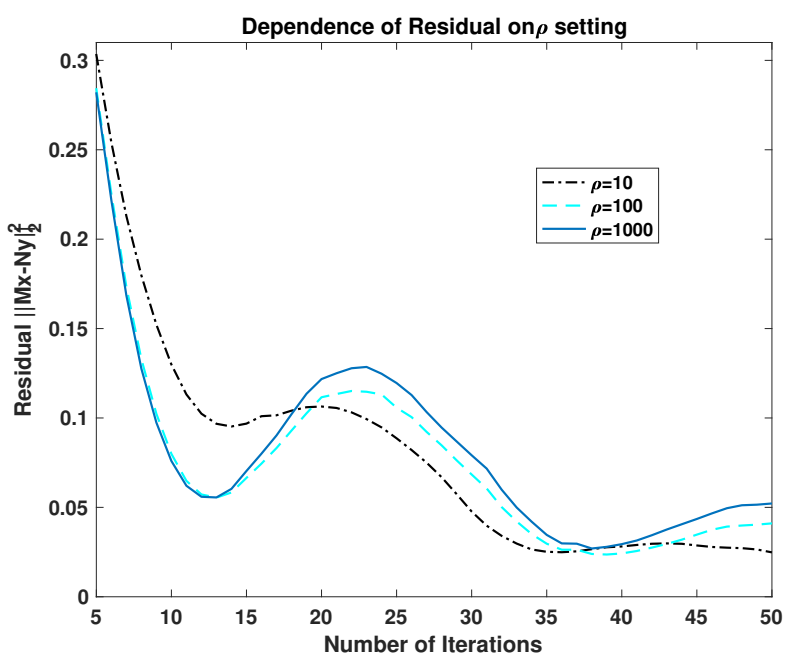

Fig. 2: Selection of $\rho$

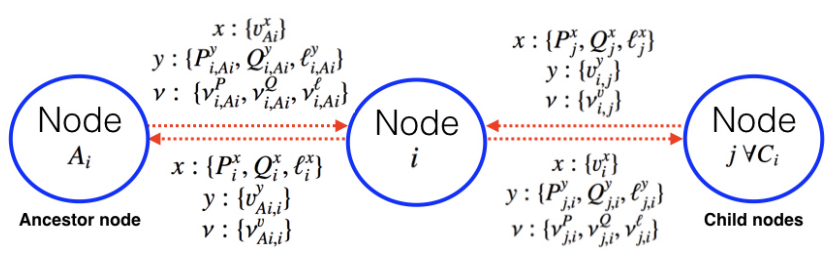

Fig. 3: Information exchange processes

To guarantee convergence of the iterative computation process, both sub-problems defined in $U 1$ and $U 2$ must be convex [26]. As $\mathcal{X}_{i}$ is not convex, $U 1$ is not convex. On the other hand, $U 2$ is convex as it is composed of convex objective and constraints. Thus, applying ADMM to the current definition of $U 1$ and $U 2$ will not guarantee convergence. In the next section, the technique by which this non-convexity is eliminated is presented.

\section{B. Eliminating Non-convexity in Sub-Problems}

The non-convex sub-problem for bus agent $i$ is $\mathcal{P}_{U 1}^{i}$ which is listed in the general form for the reader's convenience.

$$
\begin{aligned}
& \mathcal{P}_{U 1}^{i}: \underset{x_{i} \in \mathcal{X}_{i}}{\operatorname{minimize}} \frac{1}{2} x_{i}^{T}\left(A_{0}^{i}+B_{0}^{i}\right) x_{i}+C_{0}^{i T} x_{i} \\
& \text { s.t. } \frac{1}{2} x_{i}^{T} A_{1}^{i} x_{i} \leq 0, \text { where } A_{1}^{i}=2 \cdot\left[\begin{array}{cccccc}
-1 & 0 & 0 & 0 & 0 & 0 \\
0 & -1 & 0 & 0 & 0 & 0 \\
0 & 0 & 0 & \frac{1}{2} & 0 & 0 \\
0 & 0 & \frac{1}{2} & 0 & 0 & 0 \\
0 & 0 & 0 & 0 & 0 & 0 \\
0 & 0 & 0 & 0 & 0 & 0
\end{array}\right]
\end{aligned}
$$

The super-script $i$ in $\mathcal{P}_{U 1}^{i}$ will not be included henceforth for convenience and will refer to the sub-problem solved by bus agent $i$ unless otherwise mentioned. The objective is composed of quadratic and linear terms. The quadratic terms derived from $\mathcal{L}_{\rho}$ can be readily verified to be positive semi-definite implying that the objective is a convex function. The problem lies within the constraint. It is clear that $A_{1}^{i}$ is not positive semi-definite as all eigenvalues of this matrix is not nonnegative. Thus, the quadratic constraint defines a non-convex feasibility region for the $x_{i}$ variable. However, it is important to note that $\mathcal{P}_{U 1}$ is a quadratically constrained quadratic program (QCQP) with a single constraint. These problems can be solved exactly even when non-convex (i.e. non-convexity in the objective function and/or constraint) [27].

According to reference [26], even if each sub-problem defined in the ADMM process can be solved exactly, if these are non-convex, there is no guarantee of convergence of the iterative alternating direction variable update process defined in $(U 1)$ to $(U 3)$. To overcome this issue, we consider the dual of $U 1$ and establish strong duality with the corresponding primal problem via the Schur's complement and S-procedure. As such, the dual function $g_{U 1}\left(\lambda_{i}\right)$ of $\mathcal{P}_{U 1}$ is:

$$
g_{U 1}\left(\lambda_{i}\right): \underset{x_{i}}{\operatorname{minimize}} \frac{1}{2} x_{i}^{T}\left(A_{0}^{i}+B_{0}^{i}\right) x_{i}+C_{0}^{i T} x+\lambda_{i}\left(\frac{1}{2} x_{i}^{T} A_{1}^{i} x_{i}\right)
$$

where the objective is the Lagrangian $\mathcal{L}_{U 1}$ of $\mathcal{P}_{U 1}$ and $\lambda_{i}$ is the dual variable associated with quadratic inequality constraint in $\mathcal{P}_{U 1}$. First-order optimality condition is utilized to express $x_{i}$ in terms of $\lambda_{i}$ by setting the gradient of $\mathcal{L}_{U 1}$ to 0 :

$$
x_{i}=-\left(A_{0}^{i}+B_{0}^{i}+\lambda_{i} A_{1}^{i}\right)^{-1} C_{0}^{i}
$$

Substituting this expression for $x_{i}$ into $g_{U 1}$ results in the dual problem $\mathcal{D}_{U 1}$ :

$$
\begin{gathered}
\mathcal{D}_{U 1}: \underset{\lambda_{i}}{\operatorname{maximize}}-\frac{3}{2} C_{0}^{i T}\left(A_{0}^{i}+B_{0}^{i}+\lambda_{i} A_{1}^{i}\right)^{-1} C_{0}^{i} \\
\text { s.t. } \lambda_{i} \geq 0 \\
\quad A_{0}^{i}+B_{0}^{i}+\lambda_{i} A_{1}^{i} \succeq 0
\end{gathered}
$$

Constraint $D 1^{\prime}$ is imposed on dual variables that are associated with the inequality constraints in the primal problem. Constraint $D 2^{\prime}$ is necessary to ensure that there exists a finite 
solution for $x_{i}$. Problem $\mathcal{D}_{U 1}$ can now be expressed as $\mathcal{S}_{U 1}$ by applying the Schur's complement. $\mathcal{S}_{U 1}$ is a semi-definite program that is convex.

$$
\begin{aligned}
\mathcal{S}_{U 1}: & \underset{\lambda_{i}, \gamma_{i}}{\operatorname{minimize}}-\gamma_{i} \\
\text { s.t. } & \lambda \geq 0 \\
& {\left[\begin{array}{cc}
A_{0}^{i}+B_{0}^{i}+\lambda_{i} A_{1}^{i} & C_{0}^{i} \\
C_{0}^{i T} & -\gamma_{i}
\end{array}\right] \succeq 0 } \\
& A_{0}^{i}+B_{0}^{i}+\lambda_{i} A_{1}^{i} \succeq 0
\end{aligned}
$$

The dual problem is always convex [28] if the primal problem is finite. This is ensured by constraint $\left(D 2^{\prime}\right)$. Thus, the solution to every sub-problem $P_{U 1}$ can be obtained by solving for $S_{U 1}$ which is a convex problem. However, the dual solution is not always necessarily equal to the primal solution. For this to hold, strong duality must be established. When the primal problem is convex, strong duality will hold when the Slater's condition holds (i.e. the constraint set of the primal problem is non-empty). However, since $\mathcal{P}_{U 1}$ is not convex, we resort to the results established in control literature via the S-procedure to derive Theorem 1.

Theorem 1. The duality gap between $\mathcal{P}_{U 1}$ and $\mathcal{S}_{U 1}$ is zero when the constraint set of $\mathcal{P}_{U 1}$ is non-empty. This implies that the primal solution and dual solution are equal (i.e. $\mathcal{P}_{U 1}^{*}=$ $\left.\mathcal{S}_{U 1}^{*}\right)$.

Proof. The following positive semi-definite inequality is constructed using parameters from the quadratically constrained quadratic program $(\mathrm{QCQP}) \mathcal{P}_{U 1}$ :

$$
\left[\begin{array}{cc}
A_{0}+B_{0} & C_{0} \\
C_{0}^{T} & D_{0}-\gamma
\end{array}\right]+\lambda\left[\begin{array}{cc}
-A_{1} & 0 \\
0 & D_{1}
\end{array}\right] \succeq 0
$$

According to S-procedure in control literature [29], for a QCQP containing a single constraint, when there exists a $\lambda \geq 0$ that satisfies the above relation, then strong duality gap can be established with the dual of the QCQP. The expression above is in fact the constraint of the equivalent dual construction $\mathcal{S}_{U 1}$. Thus, the primal solution will be equal to the dual solution.

These transformations are utilized to define the decentralized DN coordination algorithm summarized in Alg. 1.

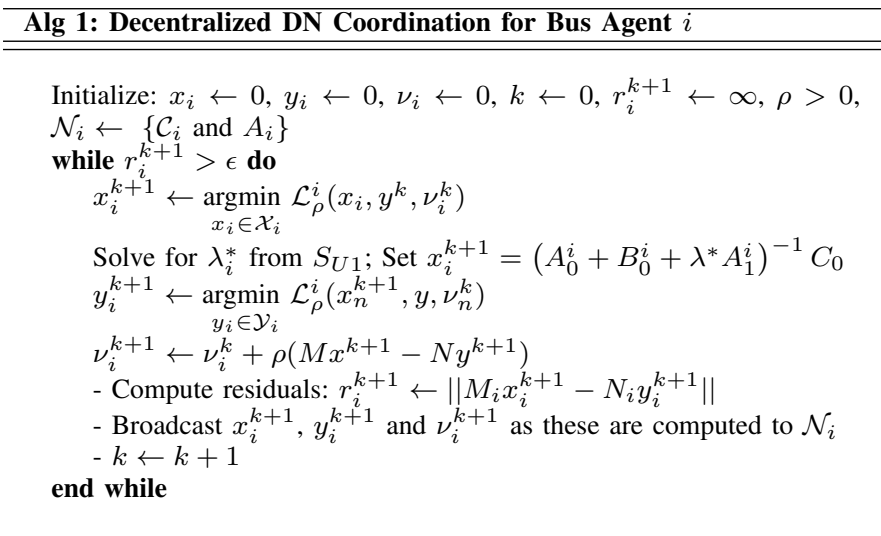

\section{Convergence Properties}

Next, we establish the convergence properties of the iterative updates made by each bus agent according to Alg 1. Specifically, we show that the system will converge to the KKT point of the original problem $\mathcal{P}_{O C}$ under one of two conditions in Theorem 2.

Theorem 2. When either one of the following conditions hold, the sub-problems solved by individual agents will converge to the KKT point of $\mathcal{P}_{O C}$.

$$
\begin{aligned}
& \text { Condition 1: } x_{i}^{k} \rightarrow y_{i j}^{k} \text { for } k \rightarrow \infty \\
& \text { Condition 2: } \nu_{i j}^{k+1}-\nu_{i j}^{k} \rightarrow 0 \text { for } k \rightarrow \infty
\end{aligned}
$$

Proof. Problem $\mathcal{P}_{O C}$ is reiterated in the following in terms of $x_{i}$ variables:

$$
\begin{aligned}
& \min _{x} \sum_{i \in \mathcal{N}}\left(\frac{1}{2} x_{i} A_{i}^{0} x_{i}+C_{i}^{0^{T}} x_{i}\right) \\
& \text { s.t. } \frac{1}{2} x_{i}^{T} A_{i}^{1} x_{i} \leq 0 ; \sum_{j \in \mathcal{N}_{i}}\left(a_{i j}^{m} x_{j}-b_{i m}\right)=0 \forall m \in m_{i} \forall i \in \mathcal{N}
\end{aligned}
$$

Each inequality constraint in the formulation above forms $\mathcal{X}_{i}$ while all $m_{i}$ equality constraints represent $\mathcal{Y}_{i}$. The Lagrangian associated with $\mathcal{P}_{O C}$ is:

$\mathcal{L}_{O C}=\sum_{i \in \mathcal{N}}\left(\frac{1}{2} x_{i}\left(A_{i}^{0}+\lambda_{i}\right) x_{i}+C_{i}^{0^{T}} x_{i}+\sum_{j \in \mathcal{N}_{i}} \sum_{m \in m_{i}} \gamma_{m}^{i}\left(a_{i j}^{m} x_{j}-b_{i m}\right)\right)$

where $\lambda_{i}$ and $\gamma_{m}^{i}$ are dual variables associated with the inequality and equality constraints respectively. The KKT conditions of $\mathcal{P}_{O C}$ for all $i \in \mathcal{N}$ are:

First-order Condition : $\left(A_{i}^{0}+\lambda_{i}^{*}\right) x_{i}^{*}+C_{i}^{0}+\sum_{j \in \mathcal{N}_{i}} \sum_{m \in m_{i}} \gamma_{m}^{i} a_{i j}^{m}=0$ Primal Feasibility : $\frac{1}{2} x_{i}^{* T} A_{i}^{1} x_{i}^{*} \leq 0 ; \sum_{j \in \mathcal{N}_{i}}\left(a_{i j}^{m} x_{j}^{*}-b_{i m}\right)=0 \forall m \in m_{i}$ Dual Feasibility : $\lambda_{i}^{*} \geq 0 ; \gamma_{m}^{i}{ }^{*} \in \mathbb{R} \forall m \in m_{i}$ Complementary Slackness : $\lambda_{i}^{*} \frac{1}{2} x_{i}^{* T} A_{i}^{1} x_{i}^{*}=0$

where $x_{i}^{*}, \lambda_{i}^{*}$ and $\gamma_{m}^{i}{ }^{*}$ are optimal primal and dual variables. In the following, it will be shown that when either Conditions 1 or 2 holds, the decomposed problems solved by each subagent will converge to the KKT point satisfying the above conditions. As such, the first-order conditions (FOCs) of $\mathcal{P}_{U 1}^{i}$ and $\mathcal{P}_{U 2}^{i}$ are:

$$
\begin{aligned}
& \text { FOC of } \mathcal{P}_{U 1}: A_{i}^{0} x_{i}+C_{i}^{0}+\rho \sum_{j \in \mathcal{N}_{i}}\left(x_{i}-y_{i j}+\frac{\nu_{i j}}{\rho}\right)+\lambda_{i} A_{i}^{1} x_{i}=0 \\
& \text { FOC of } \mathcal{P}_{U 2}:-\rho\left(x_{i}-y_{i j}\right)-\nu_{i j}+\sum_{m \in m_{i}} a_{i j}^{m} \gamma_{m}^{i}=0
\end{aligned}
$$

Due to the manner in which $\nu_{i}$ is updated, as listed in (U3), it is clear that when either condition 1 or 2 holds, the other condition will be automatically satisfied. Thus, when $k \rightarrow \infty$, the first two variables within the summation term of the FOC for $\mathcal{P}_{U 1}$ along with the first term in the FOC of $\mathcal{P}_{U 2}$ will go to 0 . Then, substituting $\nu_{i j}=\sum_{m \in m_{i}} a_{i j}^{m} \gamma_{m}^{i}$ (obtained by rearranging the remaining terms in the FOC of $\mathcal{P}_{U 2}$ ) into the FOC of $P_{U 1}$ and grouping the first and the last terms 
together in this relation, recovers the FOC condition of the original $\mathcal{P}_{O C}$. Moreover, since consensus is achieved between the $x$ and $y$ variables, the primal, dual and complementary conditions are also satisfied by the decomposition process.

\section{Discrete Control Devices}

Next, we discuss how we deal with non-convexities introduced by discrete control devices like OLTCs and shunt devices. In Eq. 14, the term $\frac{v_{i}}{t_{l}^{2}}$ is a quadratic term which introduces non-linearities in this voltage drop equation. We make a variable transformation as follows $\sum_{d=1}^{D} T_{l}^{d}=\frac{1}{t_{l}^{2}}$ where $t_{l}$ can be readily recovered from $\sum_{d=1}^{D} T_{l}^{d}$. The index of the summation $d$ denotes the $d^{\text {th }}$ discrete level in the following set: $T_{l}^{d} \in\{0.8264, \ldots, 1.23\}$ where each value is the inverse quadratic of the original values that $t_{l}$ can take as listed in Eq. 13. Thus, Eq. 14 is transformed to the following:

$$
\left(\sum_{d}^{D} T_{l}^{d}\right) v_{i}-v_{j}=2\left(r_{l} P_{i j}+x_{l} q_{i j}\right)-\left(r_{l}^{2}+x_{l}^{2}\right) \ell_{i}
$$

We assign an agent $d$ for each tap ratio which will be incharge of the following constraint:

$$
T_{l}^{d}\left(d_{\text {level }}-T_{l}\right)=0
$$

where $d_{\text {level }}$ corresponds to the tap ratio associated with $d$. This constraint forces the tap ratio agent to either activate the tap ratio or set it to 0 . This is a single non-convex constraint which forms the $x$ sub-problem for the tap ratio agent. Next, we assign an agent for the OLTC which is in-charge of the following constraints:

$$
\begin{aligned}
& 0.8264 \leq \sum_{d}^{D} T_{l}^{d} \leq 1.23 \\
& \left(\sum_{d}^{D} T_{l}^{d}\right) v_{i}-v_{j}=2\left(r_{l} P_{i j}+x_{l} q_{i j}\right)-\left(r_{l}^{2}+x_{l}^{2}\right) \ell_{i} \geq 0 \\
& \left(\sum_{d}^{D} T_{l}^{d}\right) v_{i}-v_{j}=2\left(r_{l} P_{i j}+x_{l} q_{i j}\right)-\left(r_{l}^{2}+x_{l}^{2}\right) \ell_{i} \leq 0
\end{aligned}
$$

where the first constraint enforces only one active value to be selected for the tap ratio of the OLTC, the second and third constraints represent the equality constraint listed in Eq. 19. This constraint is divided into two parts so that the $\mathrm{x}$ subproblem and the y sub-problem can be formed for the OLTC agent. The first two constraints are convex and form the $y$ subproblem and the last constraint is non-convex and forms the $x$ sub-problem. Both the tap ratio agent and the OLTC agent solve a feasibility problem and thus are associated with an objective function that can take any constant value. We have now decomposed the OLTC constraints into sub-problems for which Theorems 1 and 2 apply. Thus, discrete control devices can be readily integrated into the OPF.

\section{EXTENSION TO UNBALANCED DNS}

We extend the proposed decentralized algorithm for the unbalanced multi-phase distribution system in this section. There are various models proposed in the literature to capture the physical couplings in an unbalanced DN system. One example is the use of dq0 frame of reference (e.g. utilized in reference [30]) which transforms AC state variables (e.g. voltage and current) around a rotating frame of reference to linear variables. Imbalances in the system are accounted for by these $\mathrm{dq} 0$ variables. Although the algorithm proposed in this paper can be applied to the dq0 frame of reference, we refrain from utilizing this model in order to maintain continuity with the previous section. We extend the proposed algorithm for the unbalanced radial DN systems via the multi-phase branch-flow model introduced in reference [31]. Notations associated with this model are much more complex as variables associated with every bus and line in the $\mathrm{DN}$ are composed of up to three phases with complex components as introduced next.

Each bus and line in the DN can have up to three phases: $\phi=\{a b c\}$. If a phase does not exist for a variable, then the corresponding phase element is set to 0 . The voltage of bus $r \in \mathcal{N}$ is a complex vector composed of three components: $V_{r}=\left[V_{r}^{a} V_{r}^{b} V_{r}^{c}\right]^{T}$ where the superscripts $a, b, c \in \phi$ represent the bus voltage at each phase which is a complex variable. The current flowing on line $r \rightarrow l \in \overrightarrow{\mathcal{E}}$ is $I_{r l}=\left[\begin{array}{lll}I_{r l}^{a} & I_{r l}^{b} & I_{r l}^{c}\end{array}\right]^{T}$. The complex power injected in bus $r$ is $s_{r}=\left[\begin{array}{lll}s_{r}^{a} & s_{r}^{b} & s_{r}^{c}\end{array}\right]^{T}$. Each complex variable associated with a phase is represented using rectangular coordinates (e.g. $V_{r}^{a}=\operatorname{Re}\left\{V_{r}^{a}\right\}+\mathbf{j} \operatorname{Im}\left\{V_{r}^{a}\right\}$ where $\mathrm{Re}$ and $\mathrm{Im}$ denote real and imaginary components respectively). The complex impedance of line $(r \rightarrow l) \in \vec{E}$ is a three by three complex matrix: $z_{r l} \in \mathbb{C}^{3 \times 3}$.

System equations are presented next. The real component of power balance on bus $r$ for phase $a \in \phi$ is captured by:

$$
\begin{gathered}
\operatorname{Re}\left[\sum _ { ( q \rightarrow r ) : \vec { E } } \left[\left(V_{q}^{a}\left(I_{q r}^{a}\right)^{H}+\alpha^{2} V_{q}^{b}\left(I_{q r}^{b}\right)^{H}+\alpha V_{q}^{c}\left(I_{q r}^{c}\right)^{H}\right)-\left(z_{q r}^{a a} I_{q r}\left(I_{q r}\right)^{H}\right.\right.\right. \\
\left.\left.\left.+z_{q r}^{a b} I_{q r}^{b}\left(I_{q r}^{a}\right)^{H}+z_{q r}^{a c} I_{q r}^{c}\left(I_{q r}^{a}\right)^{H}\right)\right]+s_{r}^{a}\right]=\operatorname{Re}\left\{S_{r l}^{a}\right\}
\end{gathered}
$$

where $\alpha=e^{j 2 \pi / 3}$ and superscripts $b$ and $c$ denote the other two phases. The left hand side of the above equation represents power flowing from lines $(q \rightarrow r) \in \vec{E}$ into bus $r$ while accounting for resistive losses and power injections. The right hand side represents the power flowing out into phase $a$ of line $(r \rightarrow l) \in \vec{E}$ which is incident to bus $r$ and closer to the feeder. Since this is a radial network, bus $r$ will be connected to only one upstream bus $l$. Similarly, the imaginary counterpart of power flow on phase $a$ of bus $r$ can be derived by replacing Re with Im in the above equation. This is not a convex relation as it is an equality constraint composed of quadratic terms. Furthermore, upper limits are imposed on reverse power flows (e.g. tranformers) $(r \rightarrow l) \in \overrightarrow{\mathcal{E}}$. To account for this, we reformulate this constraint into an inequality constraint listed in Eq. 25 that accounts for this upper limit $\overline{S_{r l}^{a}}$.

$$
\begin{gathered}
\operatorname{Re}\left[\sum _ { ( q \rightarrow r ) : \vec { E } } \left[\left(V_{q}^{a}\left(I_{q r}^{a}\right)^{H}+\alpha^{2} V_{q}^{b}\left(I_{q r}^{b}\right)^{H}+\alpha V_{q}^{c}\left(I_{q r}^{c}\right)^{H}\right)-\left(z_{q r}^{a a} I_{q r}\left(I_{q r}\right)^{H}\right.\right.\right. \\
\left.\left.\left.+z_{q r}^{a b} I_{q r}^{b}\left(I_{q r}^{a}\right)^{H}+z_{q r}^{a c} I_{q r}^{c}\left(I_{q r}^{a}\right)^{H}\right)\right]+s_{r}^{a}\right] \leq \operatorname{Re}\left\{\overline{S_{r l}^{a}}\right\}
\end{gathered}
$$

Eq. 25 is still a non-convex relation as the negative quadratic terms are concave entities. and this will be dealt with in the decomposition process outlined later. The imaginary counterpart for the above constraint is: 


$$
\begin{gathered}
\operatorname{Im}\left[\sum _ { ( q \rightarrow r ) : \vec { E } } \left[\left(V_{q}^{a}\left(I_{q r}^{a}\right)^{H}+\alpha^{2} V_{q}^{b}\left(I_{q r}^{b}\right)^{H}+\alpha V_{q}^{c}\left(I_{q r}^{c}\right)^{H}\right)-\left(z_{q r}^{a a} I_{q r}\left(I_{q r}\right)^{H}\right.\right.\right. \\
\left.\left.\left.+z_{q r}^{a b} I_{q r}^{b}\left(I_{q r}^{a}\right)^{H}+z_{q r}^{a c} I_{q r}^{c}\left(I_{q r}^{a}\right)^{H}\right)\right]+s_{r}^{a}\right] \leq \operatorname{Im}\left\{S_{r l}^{\bar{a}}\right\}
\end{gathered}
$$

Next, limits on the voltage and power injection on bus $r \in \mathcal{N}$ and current flow on line $(r \rightarrow l) \in \overrightarrow{\mathcal{E}}$ are presented:

$$
\begin{aligned}
& V \leq V_{r}^{a}\left(V_{r}^{a}\right)^{H} \leq \bar{V} \forall a \in \phi \\
& s_{r}^{a}=s_{G r}^{a}-s_{L r}^{a}, s_{G r}^{a} \leq s_{G r}^{a} \leq \overline{s_{G r}^{a}} \forall a \in \phi \\
& I_{r l}^{a}\left(I_{r l}^{a}\right)^{H} \leq I_{r l}^{\bar{a}} \forall a \in \phi
\end{aligned}
$$

Eq. 27 is the voltage magnitude constraint on each phase, the second set of constraints pertain to the complex power injection in each phase, and Eq. 29 imposes upper limits on the magnitude of current flow. Constraints listed in Eq. 25 to 29 govern the underlying physical processes and limits pertaining to bus $r \in \mathcal{B}$ and line $(r \rightarrow l) \in \vec{E}$ for phase $a$. These equations apply to each one of phase $b$ and $c$ as well.

The decomposition of the power flow problem for the multiphase unbalanced DN is outlined next. This decomposition allocates two set of constraints for each agent where one constraint set is defined by a single non-convex constraint and the other is composed of convex constraints. This allows us to invoke Theorems 1 and 2. As such, we introduce three agents for each line and phase: "Re" agent, "Im" agent and "Comp" agent. The "Re" agent belonging to phase $a \in \phi$ keeps track of the local variable $\operatorname{Re}\left(I_{r l}^{a}\right)$ and is denoted as agent $\left(A_{r l}^{a}\right)^{R e}$. It maintains the perspective variables of other agents necessary to compute the relation in Eq. 25. The $x$ and $y$ variables pertaining to this agent can take values in the set $\mathcal{X}\left(A_{r l}^{a}\right)^{R e}$ and $\mathcal{Y}\left(A_{r l}^{a}\right)^{R e}$ respectively as defined in the following:

$$
\begin{aligned}
& \mathcal{X}\left(A_{r l}^{a}\right)^{R e}=\left\{\operatorname{Re}\left(I_{r l}^{a}\right)^{x}: \operatorname{Re}\left(I_{r l}^{a}\right)^{x} \in \mathbb{R}\right\} \\
& \mathcal{Y}\left(A_{r l}^{a}\right)^{R e}=\left\{\left(s_{r}^{a}\right)^{y}, V_{q}^{y}, I_{q r}^{y} \forall(q \rightarrow r) \in \vec{E}: \text { Eq. 25 }\right\}
\end{aligned}
$$

Next, the "Im" agent belonging to phase A keeps track of the local variable $\operatorname{Im}\left(I_{r l}^{a}\right)$ and is denoted as agent $\left(A_{r l}^{a}\right)^{I m}$. It maintains the perspective variables of other agents necessary to compute the relation in Eq. 25. The $x$ and $y$ variables pertaining to this agent can take values in the set $\mathcal{X}\left(A_{r l}^{a}\right)^{I m}$ and $\mathcal{Y}\left(A_{r l}^{a}\right)^{I m}$ which are defined as follows:

$$
\begin{aligned}
& \mathcal{X}\left(A_{r l}^{a}\right)^{I m}=\left\{\operatorname{Re}\left(I_{r l}^{a}\right)^{x}: \operatorname{Im}\left(I_{r l}^{a}\right)^{x} \in \mathbb{R}\right\} \\
& \mathcal{Y}\left(A_{r l}^{a}\right)^{I m}=\left\{\left(s_{r}^{a}\right)^{y}, V_{q}^{y}, I_{q r}^{y} \forall(q \rightarrow r) \in \vec{E}: \text { Eq. 26 }\right\}
\end{aligned}
$$

Finally, the "Comp" agent belonging to phase A keeps track of the magnitude of $I_{r l}^{a}$ and is denoted as agent $\left(A_{r l}^{a}\right)^{C}$. It maintains the perspectives of the real and imaginary parts of $I_{r l}$ to ensure that Eq. 29 holds. The $x$ and $y$ variables pertaining to this agent can take values in the set $\mathcal{X}\left(A_{r l}^{a}\right)^{C}$ and $\mathcal{Y}\left(A_{r l}^{a}\right)^{C}$ which are defined as follows:

$$
\begin{aligned}
& \mathcal{X}\left(A_{r l}^{a}\right)^{C}=\left\{\left|I_{r l}^{a}\right|^{x}:\left|I_{r l}^{a}\right|^{x} \in \mathbb{R}_{+}\right\} \\
& \mathcal{Y}\left(A_{r l}^{a}\right)^{C}=\left\{\operatorname{Re}\left(I_{r l}^{a}\right)^{y}, \operatorname{Im}\left(I_{r l}^{a}\right)^{y}:\left(\operatorname{Re}\left(I_{r l}^{a}\right)^{y}\right)^{2}+\left(\operatorname{Im}\left(I_{r l}^{a}\right)^{y}\right)^{2} \leq I_{r l}^{\bar{a}}\right\}
\end{aligned}
$$

It is important to note that the non-convex sets for the line agents pertain to Agent $\left(A_{r l}^{a}\right)^{R e}$ and Agent $\left(A_{r l}^{a}\right)^{I m}$ as these are composed of the power flow constraints in Eq. 25 and 26.
Next, we introduce a "Bus" agent for each bus $r \in \mathcal{N}$. This agent is denoted as $\left(A_{r}\right)$. The $x$ and $y$ variables pertaining to this agent can take values in the set $\mathcal{X}\left(A_{r}\right)$ and $\mathcal{Y}\left(A_{r}\right)$ which are defined as follows:

$$
\begin{aligned}
& \mathcal{X}\left(A_{r}\right)=\left\{V_{r}^{x}, s_{r}^{x}: \underline{V} \leq V_{r}^{a}\left(V_{r}^{a}\right)^{H}\right\} \\
& \mathcal{Y}\left(A_{r}\right)=\left\{V_{r}^{y}, s_{r}^{y}: V_{r}^{a}\left(V_{r}^{a}\right)^{H} \leq \bar{V}, \text { Eq. 28 }\right\}
\end{aligned}
$$

It is interesting to note that the $\mathcal{X}$ set is non-convex due to the lower bound constraint on quadratic voltage term whereas the $\mathcal{Y}$ set is convex.

Now that the physical constraints have been divided into the corresponding agents, the objectives for the $x$ and $y$ subproblems are defined next. The "Comp" agent $\left(A_{r l}^{a}\right)^{C}$ will have a quadratic objective of $\operatorname{Re}\left\{z_{r l}^{a}\right\}\left(\operatorname{Re}\left\{I_{r l}^{a}\right\}^{2}+\operatorname{Im}\left\{I_{r l}^{a}\right\}^{2}\right)$ in the y sub-problem to reflect the current losses in phase $a$ of line $(r \rightarrow l) \in \vec{E}$. The "Bus" agent will have a quadratic objective $C_{r}^{a} \operatorname{Re}\left\{s_{r}^{a}\right\}^{2}$ that assigns a cost for power injection into phase $a$ of bus $r$ (dictated by the generation mix in that bus). All other agents will have a constant objective function.

We have constructed a decomposition framework for the multi-phase unbalanced DN where each agent is composed of two sub-problems where one is a quadratic program with a non-convex quadratic constraint and the other problem is a convex quadratic program. These problems can be solved iteratively via the ADMM method outlined in Alg. 1 where each agent exchanges information with the agents that it maintains the perspective variables of. As these sub-problems satisfy the conditions necessary for Theorems 1 and 2, these iterative computations will converge to the KKT point of the original multi-phase unbalanced optimal power flow problem.

\section{Results}

In this section, we evaluate the practical viability of the proposed algorithm via simulations conducted using actual grid parameters in MATLAB and MATPOWER [42] platforms. The DNs implemented are IEEE 33-bus, 64-bus and 136-bus systems composed of generation systems that are added in randomly selected buses with various loading and cost parameters. The appendix contains detailed information regarding these parameters. Comparison studies are also conducted to highlight the novelty of the proposed algorithm.

\section{A. Convergence Study}

First, in the convergence study of the proposed iterative decentralized algorithm, the number of iterations necessary for the residual to reach an acceptable threshold for the 33, 64 and 136 bus systems are assessed. Results are depicted in Fig. 4a. It is clear that the initial convergence occurs very rapidly for all three systems and then the change in residual slows down as highlighted by the zoomed-in box. The 136 bus system has a higher residual and this is expected as it is composed of more bus agents than the other two systems. We have observed that all three systems converge to the residual of $\epsilon=0.01$ in 100 iterations. Each iteration $k$ involves the exchange of three sets of variables are exchanged (i.e. $x_{i}^{k}, y_{i j}^{k}$ and $\nu_{i}^{k}$ ) amongst neighbouring agents. As detailed in Sec. II, delay expected in a 


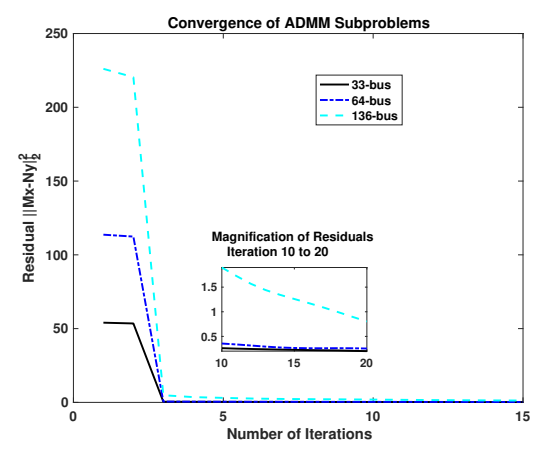

(a) Convergence rate

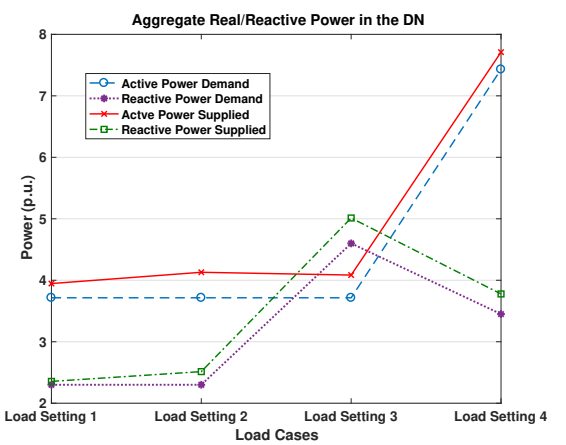

(b) Demand/supply balance

Fig. 4: System study

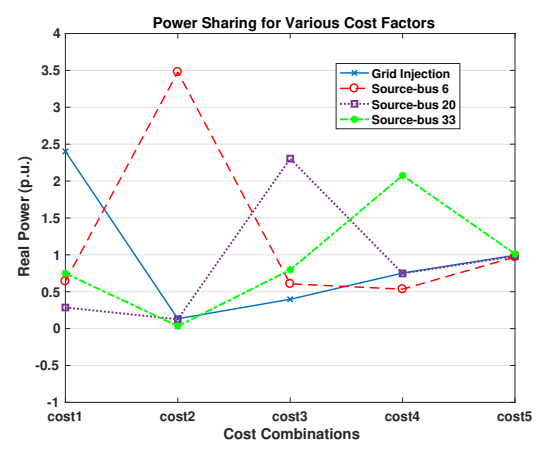

(a) Impact of cost

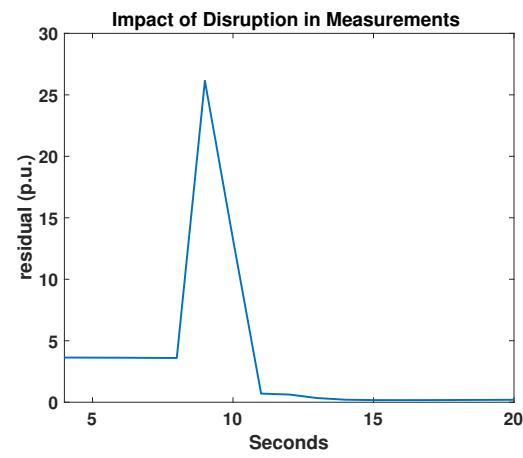

(b) Measurement interruption

Fig. 5: Power sharing

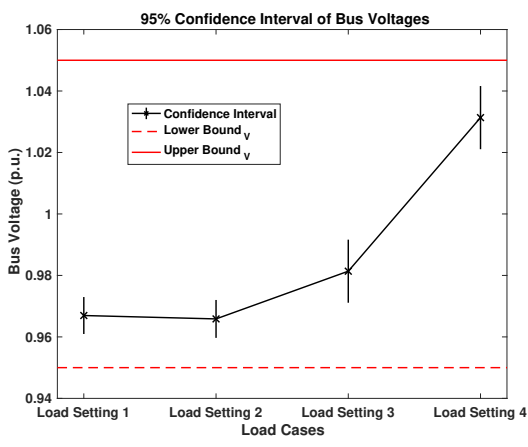

(a) Confidence interval

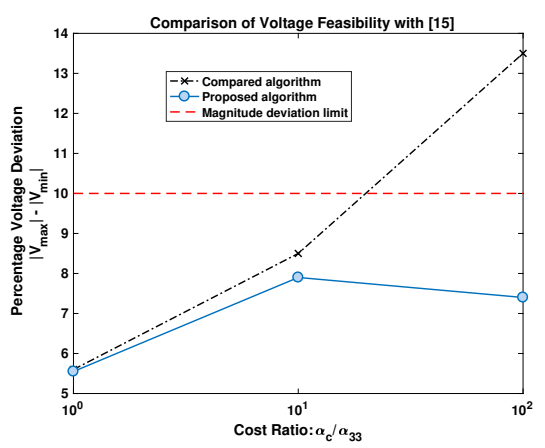

(b) Comparison

Fig. 6: Voltage feasibility

\begin{tabular}{|l|c|c|c|c|c|}
\hline & Proposed Algorithm & {$[4],[33],[34]$} & {$[10],[35],[36]$} & {$[14],[37],[38],[46]$} & {$[39],[40],[41]$} \\
\hline \hline Type of Coordination & Decentralized & Droop-based & Decentralized & Centralized & Data-driven \\
\hline Convergence & Linear & - & Linear $\mathcal{O}(n)$ & $\mathcal{O}\left(n^{k}\right)$ & - \\
\hline Application of relaxations & None & Tracking Setpoints & Convex relaxation & None & None \\
\hline Feasibility & Feasible & Feasible & Not guaranteed & Not guaranteed & Not guaranteed \\
\hline
\end{tabular}

TABLE I: Comparison of Proposed Algorithm with State-of-the-art

single hop communication is $20 \mathrm{~ms}$ in the worst case. For three sets of information exchanges, this results in $60 \mathrm{~ms}$. Adding an additional $30 \mathrm{~ms}$ for unexpected delays, we allocate $90 \mathrm{~ms}$ for each iteration. For 100 iterations (by the time the convergence occurs), this amounts to 9s. Thus, each optimization interval is 9s long and this accounts for communication delays entailed in the information exchange process. Thus, this algorithm allows for near real-time coordination of actuating power entities within the DN. In general, the convergence rate for an ADMM based algorithm is linear with respect to the number of agents $n$ in the system (i.e. $\mathrm{O}(\mathrm{n}))$ [10].

Next, in Fig. 4b, the aggregate real and reactive power injected and consumed for various load profiles is illustrated. Specifically, the overall power generated is higher than the demand in the system and this can be attributed to the losses in the power lines. Thus, it is evident that the proposed decentralized algorithm accounts for line losses as no relaxations have been applied to the underlying power flow equations. Moreover, every simulation run conducted satisfies the conditions listed in Theorem 2 (i.e. $x_{i} \rightarrow y_{i j} \forall j \in \mathcal{N}_{i}$ and $\nu_{i}^{k+1} \rightarrow \nu_{i}^{k}$ ). This implies that the algorithm converges to the associated KKT point which results in the feasibility of the power flow constraints as observed in Fig. 4b.

\section{B. Power Sharing}

The impact of the cost coefficient $A_{i}^{0}$ on power sharing amongst various power generating entities is investigated in Fig. 5a. Intuitively, the greater the cost assigned to power generated by the source in comparison to other generation systems, the lower will be the power injection by that source given that the system continues to operate within the feasible limits. Five different cost settings are utilized in the results presented in Fig. 5a. In cost combinations 1 to 4, one source is assigned a significantly lower value of $A_{i}^{0}$. In these cases, this source generates the most amount of power in comparison to other sources. The proportion of power sharing by these sources depend on how high $A_{i}^{0}$ is. Cost combination 5 renders equal power sharing amongst the three sources as the cost assigned for generation is the same for these generation systems.

\section{Missing Measurements}

When there is an interruption in the information communicated by a subset of agents, then, it is expected that the consensus gap will not close. When the agents resume regularly communicating information, the ensuing computations will result in the closing of the consensus gap. This is illustrated 
in Fig. 5b where a three-second interruption is incurred where 10 out of 33 bus agents do not communicate any information. The system is able to recover after the interruption and close the consensus gap to achieve equilibrium.

\section{System Feasibility}

Major concerns regarding the integration of power injecting sources and large loads (e.g. EVs) into the DN are overvoltage and under-voltage conditions. As the proposed algorithm accounts for the physical flows and system constraints, it is expected that the bus agents are able to maintain the operation of the system within acceptable limits. In Fig. 6a, the bus voltage magnitudes within $95 \%$ confidence interval for various system loadings are plotted. It is apparent that these voltages remain within the acceptable threshold of $1 \pm 0.05$ p.u. for all scenarios investigated. Thus, over and under-voltage conditions are averted.

\section{E. Comparison Study}

In order to highlight the novelty and contributions of the proposed algorithm, three sets of comparison studies are conducted. In the first, reference [4] that proposes optimal power sharing based on only locally obtained measurements (i.e. no communications) is implemented and the performance is quantitatively compared with the proposed algorithm. Then, in the next study, a qualitative comparison with various types of DN coordination algorithms proposed in the recent literature are presented. In the final study, we compare the performance of our algorithm with a centralized non-convex solver based on the interior point method [46].

Fig. $6 \mathrm{~b}$ illustrates the impact of not accounting for physical power flows in the coordination process. When the cost assigned to the sources are balanced, both reference [4] and our algorithm manages to keep the system within acceptable voltage thresholds. However, when the cost assigned to all sources but one is disproportionately high, the source with the lowest cost supplements all power as per the algorithm outlined in reference [4]. This results in over-voltage conditions unlike our proposal that is able to maintain local limits at a large margin. As our proposed algorithm accounts for underlying system couplings (e.g. power balance) and constraints (e.g. voltage magnitude limits), we are able to avoid this over-voltage condition by a large margin. Thus, peer-to-peer communication allows for bus agents to reach an awareness of the global conditions of the system and actuate accordingly. Neglecting power flow constraints and utilizing myopic measures can lead to system violations such as that illustrated in this figure.

Next, a comparison study of DN coordination algorithms proposed in the existing literature is presented. Attributes considered are: 1) Type of coordination (e.g. decentralized, distributed or centralized); 2) Convergence properties ( $n$ is the size of the system); 3) Application of relaxations and 4) Feasibility. In reference [32], a survey of the state-of-theart in the area of distributed and decentralized techniques for smart DNs has been presented. Amongst these, there are direct, droop-based, decentralized, centralized and data-driven techniques with which we compare our proposed algorithm in addition to centralized algorithms in Table I. A large body of existing work fall under these categories. We have included one to three references that belong to each class and we understand that these are by no means representative of these areas. However, these are composed of common attributes that facilitate discussion and comparison in the following.

References [4], [33] and [34] are droop-based algorithms where each actuating agent in the system iteratively computes local setpoints based on measurements obtained from local sensors. As only local information is used, these computations cannot account for global system conditions. Thus, actuation decisions become myopic. This is illustrated in Fig. 6b, where the droop-based algorithm proposed in reference [4] results in violations of system limits for extreme loading and cost scenarios. The next category represents decentralized algorithms such references [10], [36] and [35] that involve agents that communicate with one another to compute the optimal solution for the power flow problem. In order to overcome nonconvexities in the optimal power flow problem formulation, these proposals apply convex relaxations such as SOC and rank relaxations. These relaxations are exact under specific conditions. Hence, it is possible for the algorithm to converge to an infeasible point when these assumptions do not hold.

The third category represents centralized algorithms such as references [14], [37], [38] and [46]. These centralized solutions directly apply solvers based on heuristics to the original mixed integer non-linear program without applying any relaxations. Although these address the original problem directly, issues associated with feasibility and optimality still remain due to the non-convex nature of the problem. Furthermore, with the centralized model, actuation parameters are communicated to individual agents at every coordination interval by a central entity (e.g. utility company). This entails significant communication overheads and single point of failure issues. The final category represents an emerging class of algorithms that are data-driven and some examples include references [39], [40] and [41]. Historical and current measurements are utilized to train machine learning algorithms for computing actuation for active power elements. One major drawback pertaining to these methods is that the training data must capture a large range of states in the DN so that the actuation computed is close to that obtained from solving the optimal power flow formulation under various conditions. This entails the availability of vast volumes of labelled datasets. Our proposal overcomes the afore-mentioned shortcomings via the decentralized algorithmic design and carefully designed decomposition that allows for theoretical/practical convergence properties.

We present the third comparison study where we simulate the IEEE 33-bus system and compute optimal power flow using the algorithm presented in this paper and a centralized non-convex solver based on the interior point method [46]. The overall real and reactive power generated in the $\mathrm{DN}$ by both algorithms amount to: 3.8 p.u. and 2.3 p.u. Furthermore, the bus voltages and real power injected at each active bus (where cost is assigned for injecting active power) are similar as well and this is tabulated in Table II. It is clear that both the non-convex solver and the proposed algorithm yield the 
same solution and therefore the same objective function value. However, it is important to note that our algorithm accounts for the non-convexities and will be able to produce feasible operating points when the DN operates close to the stable limits (e.g. under congestion). Furthermore, our algorithm lends to a decentralized approach for computing the optimal power flow solution that can take advantage of an agent based system or parallel computing. As for the solution speed, both implementations result in almost instantaneous outputs and this can be attributed to the number of actuating entities present in the active DNs being considered (e.g. 136-bus DN system) not being a large value. In order to identify differences in the computational speeds of both algorithms, it is necessary to consider the asymptotic complexities of these. These are available in Table I. The proposed algorithm converges in linear time whereas the centralized algorithm using the interior point method is associated with polynomial complexity. Thus, our algorithm will be faster in the asymptotic realm.

\begin{tabular}{|c|c|c|c|c|}
\hline & \multicolumn{2}{|c|}{ Decentralized (p.u.) } & \multicolumn{2}{c|}{ Centralized (p.u.) } \\
\hline$b$ & $\left|V_{b}\right|$ & $\mathbf{P}$ & $\left|V_{b}\right|$ & $\mathbf{P}$ \\
\hline 1 & 1 & 0.94 & 1 & 0.94 \\
6 & 0.99 & 0.96 & 0.99 & 0.96 \\
20 & 1.01 & 0.93 & 1.01 & 0.93 \\
33 & 1.01 & 0.94 & 1.01 & 0.94 \\
\hline
\end{tabular}

TABLE II: Comparison with centralized non-convex solver

\section{CONCLusions}

In this paper, a novel decentralized algorithm has been presented which has been designed by leveraging on strategic decomposition, Schur's Complement, and S-procedure techniques. These allow for the construction of convex subproblems for each bus agent that can be solved exactly in a tractable manner by way of iterative information exchanges with neighbouring entities. Comprehensive theoretical analyses demonstrate the superior convergence characteristics of the proposed algorithm. Practical simulation studies conducted using realistic system parameters confirm these theoretical constructs. For future work, we intend to investigate how network reduction techniques such as Kron reduction and Zhukov's method can be utilized in the rotating dq0 frame of reference to reduce information exchanges amongst agents in the decentralized setting.

\section{ApPendix A: System PARAmeters}

In Sec. V, we have simulated IEEE 33-bus, 69-bus and 136bus systems for which the connectivity, impedance and load parameters are provided in references [43]-[45] respectively. For Fig. 4a, default parameters provided in these references are utilized for the three DN systems. For the remaining figures, we provide the parameters utilized for the 33-bus system in Figs. 7-8. The objective function utilized in the OPF is $\alpha_{i} * P_{G i}^{2}$ where $\alpha_{i}>0$ if there exists a power injecting source at bus $i$ and $\alpha_{i}=0$ otherwise. $P_{G i}$ is the amount of power generated and injected into the DN at bus $i$. As such, we have placed power injecting sources in all of these systems at buses 1, 6, 20 and 33.

\begin{tabular}{|c|c|c|c|c|c|c|c|c|}
\hline \multirow{2}{*}{ Bus No. } & \multicolumn{2}{|c|}{ Load Setting 1} & \multicolumn{2}{|c|}{ Load Setting 2} & \multicolumn{2}{|c|}{ Load Setting 3} & \multicolumn{2}{|c|}{ Load Setting 4} \\
\hline & $P_{L}(M W)$ & $Q_{L}(M V a r)$ & $P_{L}(M W)$ & $Q_{L}(M$ Var $)$ & $P_{L}(M W)$ & $Q_{L}(M$ Var $)$ & $P_{L}(M W)$ & $Q_{L}($ MVar $)$ \\
\hline 1 & & & & & & & & \\
\hline 2 & 0.10 & 0.06 & 0.10 & 0.06 & 0.10 & 0.09 & 0.20 & 0.09 \\
\hline 3 & 0.09 & 0.04 & 0.09 & 0.04 & 0.09 & 0.06 & 0.18 & 0.06 \\
\hline 4 & 0.12 & 0.08 & 0.12 & 0.08 & 0.12 & 0.12 & 0.24 & 0.12 \\
\hline 5 & 0.06 & 0.03 & 0.06 & 0.03 & 0.06 & 0.045 & 0.12 & 0.045 \\
\hline 6 & 0.06 & 0.02 & 0.06 & 0.02 & 0.06 & 0.03 & 0.12 & 0.03 \\
\hline 7 & 0.20 & 0.10 & 0.20 & 0.10 & 0.20 & 0.15 & 0.40 & 0.15 \\
\hline 8 & 0.20 & 0.10 & 0.20 & 0.10 & 0.20 & 0.15 & 0.40 & 0.15 \\
\hline 9 & 0.06 & 0.02 & 0.61 & 0.02 & 0.06 & 0.03 & 0.12 & 0.03 \\
\hline 10 & 0.06 & 0.02 & 0.06 & 0.02 & 0.06 & 0.03 & 0.12 & 0.03 \\
\hline 11 & 0.045 & 0.03 & 0.045 & 0.03 & 0.045 & 0.045 & 0.09 & 0.045 \\
\hline 12 & 0.06 & 0.035 & 0.06 & 0.035 & 0.06 & 0.053 & 0.12 & 0.053 \\
\hline 13 & 0.06 & 0.035 & 0.06 & 0.035 & 0.06 & 0.053 & 0.12 & 0.053 \\
\hline 14 & 0.12 & 0.08 & 0.06 & 0.08 & 0.12 & 0.12 & 0.24 & 0.12 \\
\hline 15 & 0.06 & 0.01 & 0.06 & 0.01 & 0.06 & 0.015 & 0.12 & 0.015 \\
\hline 16 & 0.06 & 0.02 & 0.06 & 0.02 & 0.06 & 0.03 & 0.12 & 0.03 \\
\hline 17 & 0.06 & 0.02 & 0.06 & 0.02 & 0.06 & 0.03 & 0.12 & 0.03 \\
\hline 18 & 0.09 & 0.04 & 0.09 & 0.04 & 0.09 & 0.06 & 0.18 & 0.06 \\
\hline 19 & 0.09 & 0.04 & 0.09 & 0.04 & 0.09 & 0.06 & 0.18 & 0.06 \\
\hline 20 & 0.09 & 0.04 & 0.09 & 0.04 & 0.09 & 0.06 & 0.18 & 0.06 \\
\hline 21 & 0.09 & 0.04 & 0.09 & 0.04 & 0.09 & 0.06 & 0.18 & 0.06 \\
\hline 22 & 0.09 & 0.04 & 0.09 & 0.04 & 0.09 & 0.06 & 0.18 & 0.06 \\
\hline 23 & 0.09 & 0.05 & 0.09 & 0.05 & 0.09 & 0.075 & 0.18 & 0.075 \\
\hline 24 & 0.42 & 0.20 & 0.42 & 0.20 & 0.42 & 0.30 & 0.84 & 0.30 \\
\hline 25 & 0.42 & 0.20 & 0.22 & 0.20 & 0.42 & 0.30 & 0.84 & 0.30 \\
\hline 26 & 0.06 & 0.025 & 0.06 & 0.025 & 0.06 & 0.038 & 0.12 & 0.038 \\
\hline 27 & 0.06 & 0.025 & 0.06 & 0.025 & 0.06 & 0.038 & 0.12 & 0.038 \\
\hline 28 & 0.06 & 0.02 & 0.06 & 0.02 & 0.06 & 0.03 & 0.12 & 0.03 \\
\hline 29 & 0.12 & 0.07 & 0.06 & 0.07 & 0.12 & 0.105 & 0.24 & 0.105 \\
\hline 30 & 0.20 & 0.60 & 0.10 & 0.60 & 0.20 & 0.90 & 0.40 & 0.90 \\
\hline 31 & 0.15 & 0.07 & 0.10 & 0.07 & 0.15 & 0.105 & 0.30 & 0.105 \\
\hline 32 & 0.21 & 0.10 & 0.13 & 0.10 & 0.21 & 0.15 & 0.42 & 0.15 \\
\hline 33 & 0.06 & 0.04 & 0.06 & 0.04 & 0.06 & 0.06 & 0.12 & 0.06 \\
\hline
\end{tabular}

Fig. 7: Parameters used in Figs. 3.6-3.9 for Load Settings 1-4

\begin{tabular}{|c||c|c|c|c|}
\hline Source on Bus & 1 & 6 & 20 & 33 \\
\hline \hline Cost Combo 1 & 1 & 8 & 8 & 8 \\
\hline Cost Combo 2 & 8 & 1 & 8 & 8 \\
\hline Cost Combo 3 & 8 & 8 & 1 & 8 \\
\hline Cost Combo 4 & 8 & 8 & 8 & 1 \\
\hline Cost Combo 5 & 8 & 8 & 8 & 8 \\
\hline
\end{tabular}

Fig. 8: Cost combinations (i.e. $\alpha_{i}$ )

\begin{tabular}{|c|c|}
\hline \multicolumn{2}{|l|}{ General Notation } \\
\hline $\mathcal{N}$ & Set of all buses in the DN \\
\hline$\vec{E}$ & Set of all lines in the DN \\
\hline \multicolumn{2}{|l|}{ Balanced DN } \\
\hline$z_{i j}=r_{i j}+\mathbf{j} x_{i j}$ & Complex impedance of line $(i \rightarrow j)$ \\
\hline$s_{i}=p_{i}+\mathbf{j} q_{i}$ & $\begin{array}{l}\text { Complex power injection into bus } i \text { where } \\
p_{i} \text { is the real power and } q_{i} \text { is the reactive } \\
\text { power }\end{array}$ \\
\hline$S_{i}=P_{i}+\mathbf{j} Q_{i}$ & $\begin{array}{l}\text { Complex power flow of line } i \rightarrow A_{i} \\
\text { where } A_{i} \text { is the ancestor bus that is } \\
\text { electrically closer to the feeder bus }\end{array}$ \\
\hline$S_{G i}$ & $\begin{array}{l}\text { Amount of real power and reactive power } \\
\text { generated at bus } i\end{array}$ \\
\hline$P_{G i}$ & Amount of real power generated at bus $i$ \\
\hline$S_{L i}$ & Amount of real and reactive demand at bus $i$ \\
\hline$I_{i}$ & Complex current flow of line $i \rightarrow A_{i}$ \\
\hline$\ell_{i}$ & Square magnitude of current flow of line $i \rightarrow A_{i}$ \\
\hline$V_{i}$ & Complex voltage at bus $i$ \\
\hline & Square magnitude of voltage magnitude at bus $i$ \\
\hline $\begin{array}{l}\text { Unbalanced DN } \\
\phi=\{a, b, c\}\end{array}$ & $\begin{array}{l}\text { Three phases possible for each bus and line } \\
\text { in the DN }\end{array}$ \\
\hline$z_{i j}$ & $\begin{array}{l}\text { Three-dimensional complex impedance matrix of } \\
\text { line }(i \rightarrow j)\end{array}$ \\
\hline$s_{i}=\left[\begin{array}{lll}s_{i}^{a} & s_{i}^{b} & s_{i}^{a}\end{array}\right]^{T}$ & $\begin{array}{l}\text { Complex power injection vector into } \\
\text { bus } i \text { for each phase in } \phi\end{array}$ \\
\hline$S_{i j}=\left[\begin{array}{lll}S_{i j}^{a} & S_{i j}^{b} & S_{i j}^{a}\end{array}\right]^{T}$ & Complex power flow vector of line $i \rightarrow j$ \\
\hline$I_{i j}=\left[\begin{array}{lll}I_{i j}^{a} & I_{i j}^{b} & I_{i j}^{a}\end{array}\right]^{T}$ & Complex current flow vector of line $i \rightarrow j$ \\
\hline $\begin{array}{l}V_{i}=\left[\begin{array}{lll}V_{i}^{a} & V_{i}^{b} & V_{i}^{a}\end{array}\right]^{T} \\
\text { ADMM Notation }\end{array}$ & Complex voltage at bus $i$ \\
\hline $\mathcal{X}$ & Set of local variables (i.e. $x$ variables) \\
\hline $\mathcal{Y}$ & Set of perspective variables (i.e. $y$ variables) \\
\hline$\nu$ & $\begin{array}{l}\text { Set of Lagrangian multiplies associated with } \\
\text { the consensus with } x \text { and } y \text { variables }\end{array}$ \\
\hline$\rho$ & $\begin{array}{l}\text { Positive multiplier of the augmented Lagrangian } \\
\text { term }\end{array}$ \\
\hline
\end{tabular}




\section{APPENDIX B: SYSTEM NOTATIONS}

\section{REFERENCES}

[1] H. Farhangi, "The path of the smart grid," IEEE Power Energy Magazine, vol. 8, no. 1, pp. 18-28, 2010.

[2] F. Lombardi, L. Aniello, S. D. Angelis, A. Margheri, and V. Sassone, "A Blockchain-based Infrastructure for Reliable and Cost-effective IoTaided Smart Grids," Living in the Internet of Things: Cybersecurity of the IoT, 2018

[3] C.L. Masters, "Voltage rise: the big issue when connecting embedded generation to long $11 \mathrm{kV}$ overhead lines," IET Power Engineering journal, vol. 16, no. 1, pp. 5-12, 2002.

[4] F. Chen, M. Chen, Q. Li, K. Meng, and Y. Zheng, J.M. Guerrero, and D. Abbott, "Cost-based droop schemes for economic dispatch in islanded microgrids", IEEE Transactions on Smart Grid, vol. 8, no. 1, pp. 63-74, 2017.

[5] T.L. Vandoorn, J.D.M. De Kooning, B. Meersman, and L. Vandevelde, "Review of primary control strategies for islanded microgrids with power electronic interfaces," Renewable and Sustainable Energy Reviews, vol. 19, pp. 613-628, 2013.

[6] D. E. Olivares, A. Mehrizi-Sani, A.H. Etemadi, C. Cañizares, R. Iravani, M. Kazerani, A.H. Hajimiragha, O. Gomis-Bellmunt, M. Saeedifard, R. Palma-Behnke et al., "Trends in microgrid control," IEEE Transactions on Smart Grid, vol. 5, no. 4, pp. 1905-1919, 2014.

[7] S.H. Low, "Convex relaxations of optimal power flow part I: Formulations and equivalence," IEEE Transactions on Control Network Systems, vol. 1, no. 1, pp. 15-27, 2014.

[8] H. Xu, A. Dominguez-Garcia, P. W. Sauer, "Optimal Tap Setting of Voltage Regulation Transformers Using Batch Reinforcement Learning", IEEE Transactions on Power Systems, 2019.

[9] T. Erseghe, "Distributed optimal power flow using ADMM," IEEE Transactions on Power System, vol. 29, no. 5, pp. 2370-2380, 2014.

[10] Q. Peng and S.H. Low, "Distributed algorithm for optimal power flow on a radial network," in IEEE Annual Conference on Decision and Control, pp. 167-172, 2014.

[11] P. Srikantha and D. Kundur, "Real-Time Integration of Intermittent Generation With Voltage Rise Considerations," IEEE Transactions on Sustainable Energy, vol. 8, no. 3, pp. 938-952, 2017.

[12] D. Glover, Power systems analysis and design. Stamford: Cengage Learning, 2008.

[13] M. Marzband, F. Azarinejadian, M. Savaghebi, and J.M. Guerrero, "An optimal energy management system for islanded microgrids based on multiperiod artificial bee colony combined with markov chain," IEEE Systems Journal, vol. 11, no. 3, pp. 1712-1722, 2017.

[14] M. Ross, C. Abbey, F. Bouffard, and G. Jos, "Multiobjective optimization dispatch for microgrids with a high penetration of renewable generation," IEEE Transaction on Sustainable Energy, vol. 6, no. 4, pp. 1306-1314, 2015

[15] T. Erseghe, "Distributed Optimal Power Flow Using ADMM," IEEE Transactions on Power Systems, vol. 29, no. 5, pp. 2370-2380, 2014.

[16] M. Mallick and P. Srikantha, "Optimal Decentralized Microgrid Coordination via the Schur's Complement and S-Procedure", IEEE Transactions on Smart Grid, 2019. (IEEE Early access article)

[17] Future of the Grid, Gridwise Alliance. [Online]. Available at: http://energy.gov/oe/downloads/ future-grid-evolving-meet-america-s-needs-december-2014. [Accessed: 21-Jan-2016].

[18] F. Dorfler, and F. Bullo, " Kron Reduction of Graphs With Applications to Electrical Networks", IEEE Transactions on Circuits and Systems, vol. 60, no. 1, pp. 150-163, 2013.

[19] A. Von Meier, D. Culler, A. McEachern, R. Arghandeh, "Microsynchrophasors for Distribution Systems", IEEE Innovative Smart Grid Technologies, 2014.

[20] E. Bou-Harb, C. Fachkha, M. Pourzandi, M. Debbabi and C. Assi, "Communication security for smart grid distribution networks", IEEE Communications Magazine, vol. 51, no. 1, pp. 42-49, 2013.

[21] "EcoGrid EU," EcoGrid EU. [Online]. Available: http://www.euecogrid.net/. [Accessed: 30-Mar-2020].

[22] T. Weibel, "UMassTraceRepository," Smart - UMass Trace Repository. [Online]. Available: http://traces.cs.umass.edu/index.php/Smart/Smart/. [Accessed: 30-Mar-2020].

[23] B. Krupanek and R. Bogacz, "Investigations of transmission delays in ZigBee networks", Politechnika Slaska, Instytut Metrologii, Elektroniki i Automatyki, vol. 1, no. 2, 2014.
[24] K. Orwig et al, "Recent Trends in Variable Generation Forecasting and Its Value to the Power System", IEEE Transactions on Sustainable Energy, vol. 6, no. 3, pp. 924-933, 2014.

[25] J. A. Taylor. Convex optimization of power systems. Cambridge, UK: Cambridge University Press, 2015.

[26] S. Boyd, N. Parikh, E. Chu, B. Peleato, and J. Eckstein, "Distributed optimization and statistical learning via the alternating direction method of multipliers," Foundations and Trends in Machine Learning, vol. 3, no. 1, pp. 1-122, 2011

[27] K. Huang and N. D. Sidiropoulos, "Consensus-ADMM for general quadratically constrained quadratic programming," IEEE Transactions on Signal Processing, vol. 64, no. 20, pp. 5297-5310, 2016.

[28] S. Boyd and L. Vandenberghe. Convex Optimization . Cambridge, UK: Cambridge University Press, 2004.

[29] U. T. Jonsson. (2001). "A Lecture on the S-procedure [Online]. Available: https://people.kth.se/ uj/5B5746/Lecture.ps.

[30] A.H. Etemadi, E.J. Davison, and R. Iravani, "A decentralized robust control strategy for multi-der microgrids-part I: Fundamental concepts," IEEE Transactions on Power Delivery, vol. 27, no. 4, pp. 1843-1853, Oct. 2012.

[31] L. Gan and S. H. Low, "Convex relaxations and linear approximation for optimal power flow in multiphase radial networks," Power Systems Computation Conference, 2014

[32] K. E. Antoniadou-Plytaria, I. N. Kouveliotis-Lysikatos, P. S. Georgilakis, and N. D. Hatziargyriou,"Distributed and Decentralized Voltage Control of Smart Distribution Networks: Models, Methods, and Future Research", IEEE Transactions on Smart Grid, vol. 8, no. 6, pp. 2999 3008, 2017.

[33] F. Olivier, P. Aristidou, D. Ernst, and T. Cutsem, "Active Management of Low-Voltage Networks for Mitigating Overvoltages Due to Photovoltaic Units", IEEE Transactions on Smart Grid, vol. 7, no. 2, pp. 926-936, 2016.

[34] J. Schiffer, T. Seel, J. Raisch, T. Sezi, "Voltage Stability and Reactive Power Sharing in Inverter-Based Microgrids With Consensus-Based Distributed Voltage Control", IEEE Transactions on Control Systems Technology, vol. 24, no. 1, pp. 96-109, 2016.

[35] B. Zhang, et al, "An Optimal and Distributed Method for Voltage Regulation in Power Distribution Systems", IEEE Transactions on Power Systems, vol. 30, no. 4, pp. 1714-1726, 2015.

[36] E. Dallanese, H. Zhu, and G.B. Giannakis, "Distributed optimal power flow for smart microgrids," IEEE Transactions on Smart Grid, vol. 4, no. 3, pp. 1464-1475, 2013.

[37] M. Shaheen et al, "Optimal Power Flow of Power Systems Including Distributed Generation Units Using Sunflower Optimization Algorithm", IEEE Access, vol. 7, pp. 109289-109300, 2019.

[38] C. Ye, and M. Huang, "Multi-Objective Optimal Power Flow Considering Transient Stability Based on Parallel NSGA-II", IEEE Transactions on Power Systems, vol. 30, no. 2, pp. 857-866, 2014.

[39] S. Karagiannopoulos, et. al, "Data-Driven Local Control Design for Active Distribution Grids Using Off-Line Optimal Power Flow and Machine Learning Techniques", IEEE Transactions on Smart Grid, vol. 10, no. 6, 2019.

[40] R. Dobbe et al, "Towards Distributed Energy Services: Decentralizing Optimal Power Flow with Machine Learning". IEEE Transactions on Smart Grid, 2019. (Early Access)

[41] A. Ouammi et al, "Coordinated Model Predictive-Based Power Flows Control in a Cooperative Network of Smart Microgrids", IEEE Transactions on Smart Grid, vol. 6, no. 5, 2015.

[42] R. D. Zimmerman, C. E. Murillo-Sanchez, and R. J. Thomas, "MATPOWER: Steady-State Operations, Planning and Analysis Tools for Power Systems Research and Education," IEEE Transactions on Power Systems, vol. 26, no. 1, pp. 12-19, 2011

[43] M. E. Baran, and F. Wu, "Network Reconfiguration in Distribution Systems for Loss Reduction and Load Balancing", IEEE Transactions on Power Delivery, vol. 4, no. 2, pp. 1401- 1407, 1989.

[44] M. E. Baran, and F. Wu, "Optimal Capacitor Placement on Radial Distribution Systems", IEEE Transactions on Power Delivery, vol. 4, no. 1, pp. 725-734, 1989.

[45] J. Mantovani, F. Casari, and R. Romero, "Reconfiguracao De Sistemas De Distribuicao Radiaisutilizando O Criterio De Queda De Tensao", SBA Controle and Automacao, vol. 11, no. 3, pp. 150-159, 2000.

[46] H. Y. Benson, "Mixed integer nonlinear programming using interiorpoint methods," Optimization Methods and Software, vol. 26, no. 6, pp. 911- 931, 2011. 


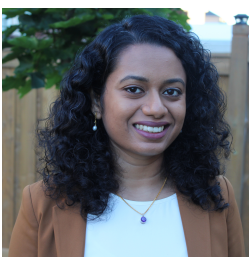

Pirathayini Srikantha is currently an Assistant Professor in the Department of Electrical Engineering and Computer Science at York University. She received her B.A.Sc. degree in Systems Design Engineering from the University of Waterloo in 2009 and her M.A.Sc. degree in Electrical and Computer Engineering from the same institute in 2013. She obtained her Ph.D. degree from The Edward S. Rogers Sr. Department of Electrical and Computer Engineering at the University of Toronto in 2017. She is currently serving as an Associate Editor for the IEEE Transactions on Smart Grid journal. She is a certified Professional Engineer (P.Eng.) in Ontario. Her main research interests are in the areas of large-scale optimization and distributed control for enabling adaptive, sustainable and resilient power grid operations. Her work has been published in premier smart grid journal and conference venues. Her research efforts have received recognitions that include the best paper award (IEEE Smart Grid Communications) and runner-up best poster award (ACM Women in Computing).

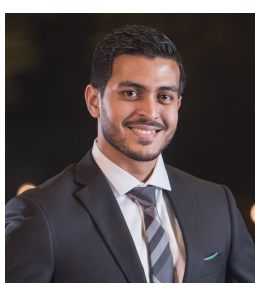

Mithun Mallick received his M.E.Sc. degree from the Electrical and Computer Engineering department at Western University. He received his B.Sc. degree from the Electrical \& Electronic Engineering department of Bangladesh University of Engineering \& Technology, Dhaka, Bangladesh in 2017. He is currently an Electrical Engineer with Phoventus Inc. $\mathrm{He}$ is also a student member of the IEEE Power $\&$ Energy Society. His research interests lie in the area of optimization, protection and control of microgrids, smart grids, renewable energy systems and large-scale power systems. He is also keen in exploring the applications of various numerical methods and game theory in power systems. 